\title{
Large Extracellular Vesicles Can be Characterised by Multiplex Labelling Using Imaging Flow Cytometry
}

\author{
Suzanne M Johnson ${ }^{1, *(\mathbb{C}}$, Antonia Banyard ${ }^{2}$, Christopher Smith ${ }^{1}$, Aleksandr Mironov ${ }^{3}$ and \\ Martin G. McCabe 1,4 \\ 1 Children's Cancer Group, Division of Cancer Sciences, School of Medical Sciences, Faculty of Biology \\ Medicine and Health, University of Manchester, Oglesby Cancer Research Building, Manchester Academic \\ Health Science Centre, Manchester Cancer Research Centre, Manchester M20 4GJ, UK; \\ Christopher.Smith-5@manchester.ac.uk (C.S.); Martin.McCabe@manchester.ac.uk (M.G.M.) \\ 2 Flow Cytometry Core Facility, Cancer Research UK Manchester Institute, University of Manchester, \\ Alderley Park, Macclesfield, UK; Antonia.Banyard@manchester.ac.uk \\ 3 Electron Microscopy Core Facility, School of Biological Sciences, Faculty of Biology Medicine and Health, \\ University of Manchester, Manchester M13 9PT, UK; Aleksandr.Mironov@manchester.ac.uk \\ 4 Manchester Children's Brain Tumour Research Network Royal Manchester Children's Hospital, \\ Manchester M13 9WL, UK \\ * Correspondence: suzanne.johnson@manchester.ac.uk
}

Received: 23 September 2020; Accepted: 16 November 2020; Published: 18 November 2020

\begin{abstract}
Extracellular vesicles (EVs) are heterogeneous in size (30 nm-10 $\mu \mathrm{m})$, content (lipid, RNA, DNA, protein), and potential function(s). Many isolation techniques routinely discard the large EVs at the early stages of small EV or exosome isolation protocols. We describe here a standardised method to isolate large EVs from medulloblastoma cells and examine EV marker expression and diameter using imaging flow cytometry. Our approach permits the characterisation of each large EVs as an individual event, decorated with multiple fluorescently conjugated markers with the added advantage of visualising each event to ensure robust gating strategies are applied. Methods: We describe step-wise isolation and characterisation of a subset of large EVs from the medulloblastoma cell line UW228-2 assessed by fluorescent light microscopy, transmission electron microscopy (TEM) and tunable resistance pulse sensing (TRPS). Viability of parent cells was assessed by Annexin V exposure by flow cytometry. Imaging flow cytometry (Imagestream Mark II) identified EVs by direct fluorescent membrane labelling with Cell Mask Orange (CMO) in conjunction with EV markers. A stringent gating algorithm based on side scatter and fluorescence intensity was applied and expression of EV markers CD63, CD9 and LAMP 1 assessed. Results: UW228-2 cells prolifically release EVs of up to $6 \mu \mathrm{m}$. We show that the Imagestream Mark II imaging flow cytometer allows robust and reproducible analysis of large EVs, including assessment of diameter. We also demonstrate a correlation between increasing EV size and co-expression of markers screened. Conclusions: We have developed a labelling and stringent gating strategy which is able to explore EV marker expression (CD63, CD9, and LAMP1) on individual EVs within a widely heterogeneous population. Taken together, data presented here strongly support the value of exploring large EVs in clinical samples for potential biomarkers, useful in diagnostic screening and disease monitoring.
\end{abstract}

Keywords: extracellular vesicles; imaging flow cytometry; biomarker reservoirs; cancer diagnostics; disease monitoring; large EVs

\section{Introduction}

The term extracellular vesicle (EV) refers to particles released from cells which are delimited by a lipid bilayer, but do not contain a nucleus [1]. EVs are heterogeneous in biogenesis [2], size, and content. 
They range in size from $30 \mathrm{~nm}$ exosomes [3] to oncosomes up to $10 \mu \mathrm{M}$ [4], and contain cargo of all biomolecule categories [5]. Attempts to categorise EVs primarily by surface marker expression have been confounded by the recognition that many of the markers previously considered to be subset- or derivation-specific, are actually present on multiple or even all classes of EVs [6]. Delineation and characterisation of specific EV subsets is an essential goal to achieve a better understanding of EV biology [7], yet there are no techniques that accurately quantify EVs across the full EV size range, or combine quantification with the ability to screen for EV marker expression or EV content.

Small EVs are characteristically isolated by high-speed centrifugation at 100,000 $\times g$ [8], and include both EVs derived intracellularly from late endosomes and released by exocytosis (exosomes), and other small EVs not derived from endosomes (3). Multiple commercial solutions exist for the isolation of exosomes from a variety of biological fluids including tissue culture supernatant, plasma, and urine. In contrast, there are no commercially available solutions for the isolation of large EVs. As a result, isolation methods vary, and knowledge of large EV content and function in biological samples is relatively lacking. Large EVs, defined as $>200 \mathrm{~nm}$ by recent guidelines set out by the International Society of Extracellular Vesicles [1], include cancer cell-derived oncosomes, dead cell-derived apoptotic bodies and platelets, and are visible by light microscopy [9]. In published literature, EVs larger than $1 \mu \mathrm{m}$ have historically been assumed to be apoptotic bodies [10]. However, we and others [4] have demonstrated that viable cell cultures produce large EVs which do not have the ultrastructural features reminiscent of fragments of apoptotic cells.

EVs are released by all cells providing an efficient mechanism of cell to cell communication [11]. Increasing evidence points to key roles for EVs in cancer diagnosis, prognostication, and surveillance of cancer [12]. Large EVs from prostate cancer cells were shown to contain tumour-specific biomarkers [4,13] and mediate intercellular transfer of bioactive molecules including miRNA [14]. We previously reported a population of large EVs released by leukaemic cells which were actin-rich and contained intact organelles [15]. These large EVs could be internalised by normal stromal cells and induced a switch in the preferred metabolic pathway of the recipient cells [9]. Additionally, we found that leukaemia-derived EVs expressed a surface marker indicative of their parent cell (CD19) and could be detected in the peripheral blood of murine models and patient bone marrow plasma [9]. Taken together, our previous work and existing large EV literature suggest that large EVs, often discarded in techniques to isolate smaller EVs and exosomes, could be considered as extensive reservoirs of biomolecules useful to study EV biogenesis and function, and to identify clinically relevant biomarkers for disease detection and treatment monitoring [16,17].

The principle advantage of characterising large EVs as single events by imaging flow cytometry is the potential for simultaneously identifying parent cell, EV, and tumour markers. We report for the first time a characterisation of size distribution and EV marker expression in this heterogeneous EV population, undertaken in accordance with the most recent international consensus guidelines for EV research from the International Society of Extracellular Vesicles [1].

In this proof of concept study, we set out to: (1) highlight the abundance of large EVs produced by cells derived from the malignant brain tumour medulloblastoma in vitro; (2) describe variations in the expression of established EV markers in the large EV population; (3) describe how the Imagestream (ISX) can address sample heterogeneity by facilitating high throughput, single event EV analyses. We describe a protocol to isolate intact large EVs without cell contamination, from cells growing in serum-free medium, using gravity flow filtration combined with low-speed centrifugation. Our data show the breadth of heterogeneity in the size and marker expression of large EVs isolated from a single cell line and serves to highlight the importance of sample purity, isolation techniques and experimental controls as we seek to identify tumour-specific EV markers for use in the clinic. 


\section{Results}

Large EVs from the medulloblastoma cell line UW228-2 are visible with light microscopy, can be isolated from viable, serum-free cell culture supernatant with intact membranes, and contain a polymerised actin cytoskeleton. A proportion of these large EVs express reported EV markers.

In this study the large EV isolation SOP was applied to the SHH-driven medulloblastoma cell line UW228-2. In accordance with the recently published international consensus MISEV2018 guidelines [1], we demonstrated the existence and membrane integrity of large EVs using both light and electron microscopy (Figure 1A,B). The pan-EV marker CD63 was expressed by parent medulloblastoma cells and a proportion of large EVs in viable cultures (Figure 1A). Large EVs also exhibited an active cytoskeleton indicated by the polymerised actin marker Phalloidin, but did not stain for DAPI, indicating that they did not contain nuclear double-stranded DNA. EV membrane integrity, size, and intra-vesicle content was examined by transmission electron microscopy (TEM) (Figure 1B). Isolated EVs were spiked with medulloblastoma parent cells for comparison and adhered to ACLAR film coated with CellTak prior to TEM. Serial sections demonstrated that the EVs had a limiting membrane, internal organelles but no nucleus, and were independent from cells. By contrast, cells had internal organelles including a nucleus and cytoplasmic protrusions indicative of filopodia.

Cells were grown in serum-free medium for $24 \mathrm{~h}$ prior to EV isolation, staining, and analysis. Experimental conditions were optimised to eliminate false positive membrane labelling or bovine EV contamination [18]. To assess whether growth in serum-free medium resulted in increased cell apoptosis, phosphatidyl serine exposure on the surface of cells was examined. Figure $1 \mathrm{C}$ indicates the quadrant gating strategy applied using positive controls and the comparison of cells grown in standard culture versus serum-free conditions. Imaging flow cytometry was also performed to visualise Annexin V/PI staining on parent cells (Figure 1D). In triplicate experiments, there was no significant difference between the viability of cells cultured in complete or serum-free media (Figure 1E). Figure 1F provides a workflow for the isolation and characterisation of large EVs used in this study. At the time of each EV isolation, total parent cell count and viability was assessed using trypan blue exclusion and found to be $7.9 \times 10^{6}$ cells $\left(98 \%\right.$ viable), $8.3 \times 10^{6}$ cells $\left(99 \%\right.$ viable), and $6.8 \times 10^{6}$ cells ( $98 \%$ viable).
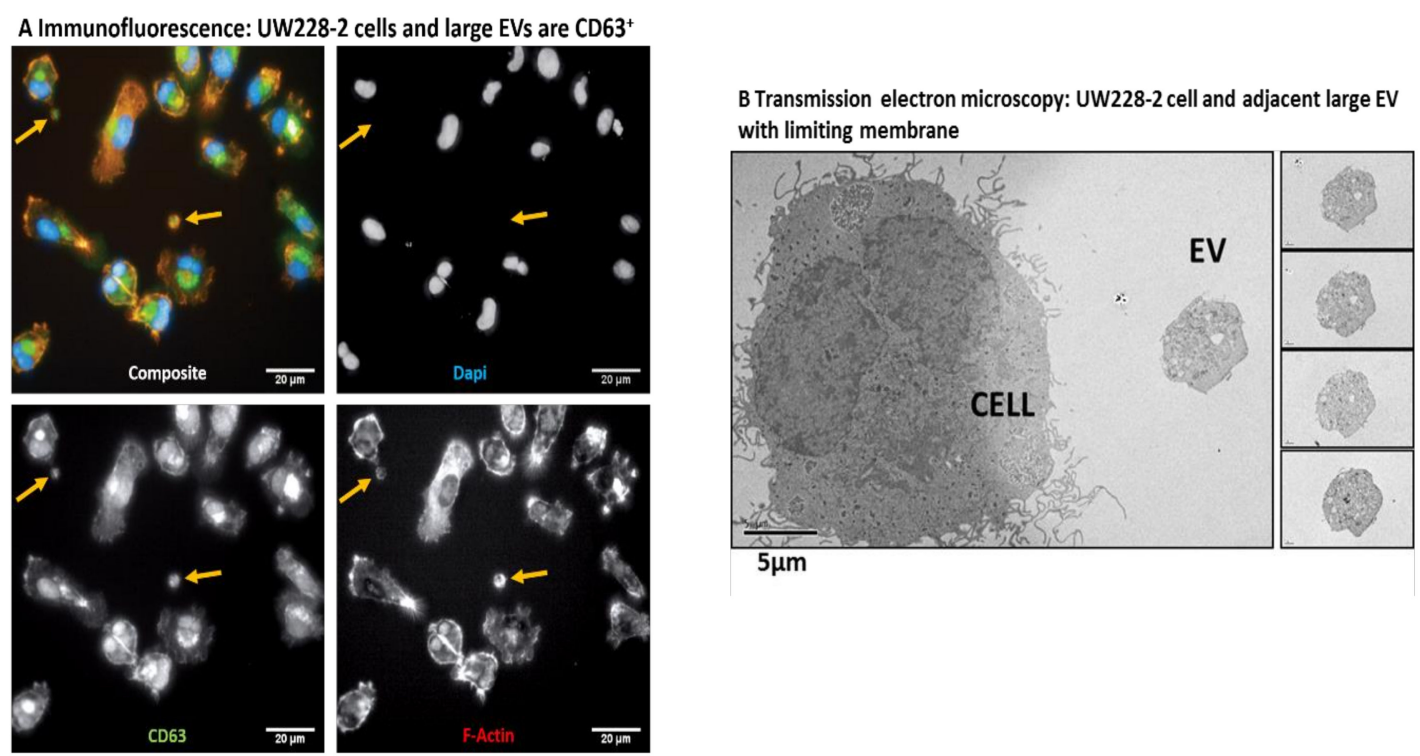

Figure 1. Cont. 
C Flow cytometry: Cell viability Annexin V/PI assessment

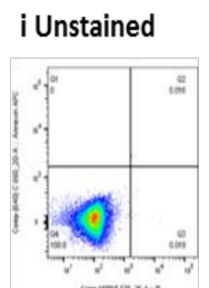

ii Etoposide $(200 \mu \mathrm{M})$

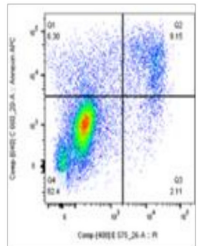

iii Heated $\left(56^{\circ} \mathrm{C}\right)$

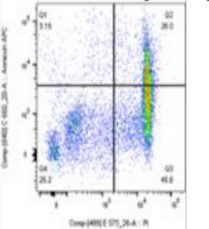

iv Complete Media

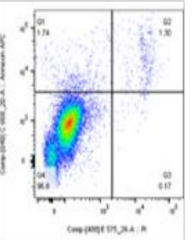

v Serum Free Media

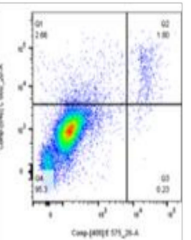

D ISX analysis: Annexin V/PI assessment in cells

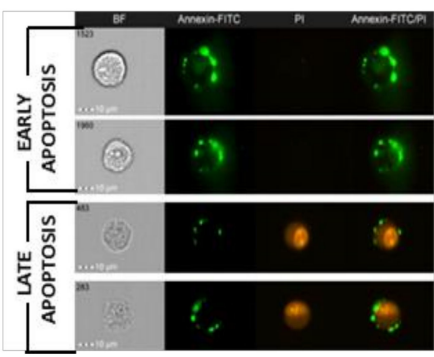

E Cell viability prior to EV isolation

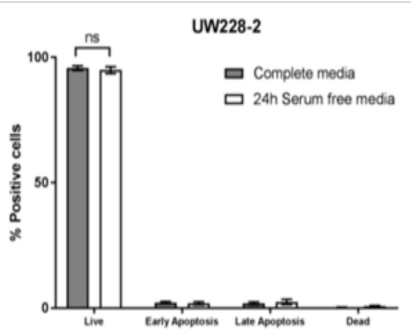

\section{F Large $\mathrm{EV}$ isolation and characterisation}

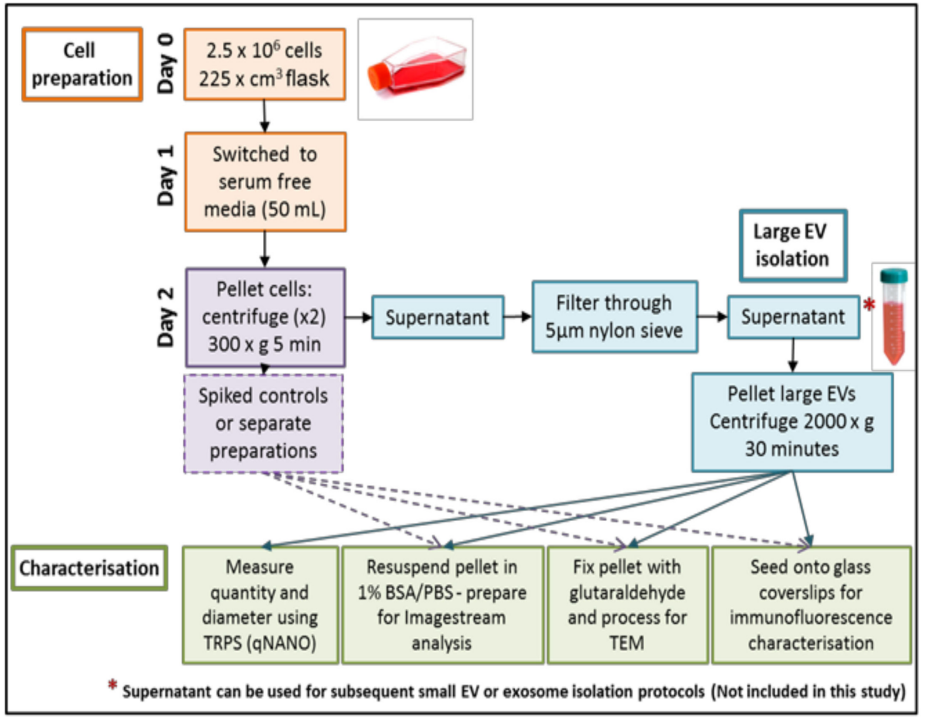

Figure 1. Immunofluorescence: UW228-2 cells and large extracellular vesicles (EVs) are CD63 ${ }^{+}$. Medulloblastoma cell lines produce large extracellular vesicles (EVs) which can be harvested from serum-free conditioned media after $24 \mathrm{~h}$. (A) Medulloblastoma cells produce EVs in vitro which contain polymerised actin and CD63, but no nucleus. The medulloblastoma cell line UW228-2 was cultured on glass bottom plates for $24 \mathrm{~h}$; fixed and probed for the tetraspanin and EV marker CD63 (FITC—green). Polymerised actin was labelled using Alexa Fluor 555 phalloidin (f-ACTIN—red). Nuclei were counterstained with DAPI (DAPI—blue). Images of large EVs (yellow arrows) and cells were captured using the Perkin Elmer Operetta system at $\times 40$ magnification. The individual images were captured in black and white using the appropriate emission filters and a coloured composite image created using Columbus software. Scale bar represents $20 \mu \mathrm{M}$. (B) Transmission electron microscopy: UW228-2 cell and adjacent large EV with limiting membrane. Large EVs have a limiting membrane, contain organelles but no nucleus and are $<6 \mu \mathrm{m}$ in size. Cells and EVs were applied to Poly-D-lysine coated ACLAR film and fixed with glutaraldehyde before processing for Transmission Electron 
Microscopy. Serial sections were taken to assess sample purity, EV membrane integrity, size, and content comparative to the parent cell. Images were captured using a Biotwin Philips TECNAI G2 microscope at $\times 1900$ magnification. Scale bar represents $5 \mu \mathrm{M}$. Left panel shows a cell and a large EV. Right panel: serial sections taken through the entire EV (4 images shown). The wide-field image shows an EV of $5.5 \mu \mathrm{M}$ diameter at its central plane of focus, alongside a parent medulloblastoma cell of $18 \mu \mathrm{M}$. (C) Flow cytometry: Cell viability Annexin V/PI assessment. Cell apoptosis was assessed by flow cytometry. Unstained cells (i) and cells treated with $200 \mu \mathrm{M}$ Etoposide (ii; Annexin V positive gate) or heated (iii; PI positive gate) provided positive controls which were used to assign accurate quadrant gates to bivariate scatter plots showing PI versus Annexin V APC. Live cells appear in Q4, Annexin only in Q2 (Early apoptosis), dual labelled in Q3 (Late apoptosis) and PI only (Dead cells). UW228-2 cells were cultured for $24 \mathrm{~h}$ in complete media (iv; DMEM with 10\% FBS) or serum-free media (v; DMEM only). Representative plots of triplicate experiments shown. (D) Imagestream (ISX) analysis: Annexin $\mathrm{V} / \mathrm{PI}$ assessment in cells. Imaging flow cytometry allows visual distinction between early and late stages of apoptosis. Cells in standard culture conditions were labelled using an Annexin FITC/PI kit and examined using the Imagestream (ISX) Mark II. Images were captured at x60 magnification and representative gallery images demonstrate Annexin V FITC only membrane labelling, indicating early apoptosis (upper panels) and dual Annexin V FITC/PI labelling, indicating late apoptosis (lower panels). (E) Cell viability prior to EV isolation. UW228-2 cells can be cultured in serum-free conditions for $24 \mathrm{~h}$ with no loss in viability. No difference in viability could be seen between culture conditions: Percentage unlabelled cells (live), Annexin V positive only (Early apoptosis), Annexin V and PI positive (Late Apoptosis) or PI positive only (DEAD) after $24 \mathrm{~h}$ in complete (grey bars) or serum-free medium (open bars). (F) Large EV isolation and characterisation. EV isolation protocol from cultured cells was optimised to harvest large EVs with minimal processing. Cells were seeded into large flasks $\left(225 \mathrm{~cm}^{3}\right)$ and allowed to adhere. Media was changed after $24 \mathrm{~h}$ for $50 \mathrm{~mL}$ serum-free DMEM and cells cultured at $37^{\circ} \mathrm{C}$ for a further $24 \mathrm{~h}$. Trypsinised cells were collected by 2 successive centrifugation steps at $300 \times g 5$ min keeping the supernatant each time. EV containing supernatant was filtered using a double layered $5 \mu \mathrm{m}$ pore nylon membrane by gravity. Filtered, cell-free supernatant was centrifuged at $2000 \times g$ for 30 min using a bench top centrifuge with swing out buckets. The resulting cell and EV pellets were processed appropriately for the downstream technique: For flow cytometry cells were processed and analysed separately, whilst cells were spiked into wells for direct comparison by microscopy.

\subsection{EVs are Highly Heterogeneous and Differentially Express EV Markers}

A reliable fluorescence marker was essential to demarcate EVs from background scatter events. Cell mask orange (CMO) is a fluorescent plasma membrane label composed of amphipathic molecules comprising a lipophilic moiety for membrane loading and a negatively charged hydrophilic dye for anchoring of the probe in the plasma membrane. We performed a titration using the parent cells with serial dilutions of the dye in serum-free media ranging from 1 in $1000(5 \mu \mathrm{g} / \mathrm{mL})$ to 1 in $100,000(50 \mathrm{ng} / \mathrm{mL}$ ) (Figure 2A) and found that $2.5 \mu \mathrm{g} / \mathrm{mL}$ (1 in 2000) was an optimal concentration providing high median fluorescence intensity without saturation. Using the Imagestream Mark II (ISX) we confirmed that the final concentration of CMO labelled EVs in a typical preparation did not result in coincidence events or swarm which could lead to false positive results when looking at multi-colour labelling. Serial dilutions of CMO labelled EVs showed a linear decrease in objects per $\mathrm{mL}$ (Figure $2 \mathrm{~B}(\mathrm{i})$ ) with an increasing dilution factor, whilst the fluorescence intensity remained stable across the dilutions (Figure 2B(ii)). To facilitate reproducible reporting across experiments and to offer a means to standardise EV measurements, we determined the minimum MESF value for $\mathrm{CMO}+$ events which would distinguish between unstained and CMO labelled EVs using our staining protocol, as described elsewhere [19]. Figure 2C shows a representative bivariate plot (i) of the low, medium-low, medium-high, and high fluorescence bead populations. The histogram (ii) was used to determine the MFI of each peak and log fluorescence intensities were converted to MESF values using information supplied by the manufacturer (iii). Linear regression of log MFI versus log MESF 
(iv) was used to calculate the MESF corresponding to the minimum fluorescence intensity of events within the designated $\mathrm{CMO}+$ gate as follows. The maximum MESF values of the unstained EVs in each experiment were: Replicate 1: $271.42=$ MESF 48.23; Replicate 2: 271.0 = MESF 49.27; Replicate 3: $272.88=$ MESF 42.48. Therefore, to set a standardised lower threshold of detection which could be used across replicate experiments we assigned a lower MESF threshold for $\mathrm{CMO}^{+}$events of 50 (Example shown in Figure $2 \mathrm{C}(\mathrm{v})$ ). We therefore report here $\mathrm{CMO}^{+}$events as number of events $>\mathrm{MESF} 50$.

Previous reports have identified that lipid dye aggregates can mimic EVs when using fluorescence lipid markers [20]. We used Triton-X 100 treatment [21] of fully labeled EVs to disrupt EV signals demonstrated by a loss of CMO + events (Figure 2D(i)). Figure 2D(ii) shows an overlay of unstained EVs, fully stained EVs and fully stained EVs treated with Triton-X 100. The MFI of the post-Triton-X 100 treated sample is reduced to the level of the US-EVs.

A.

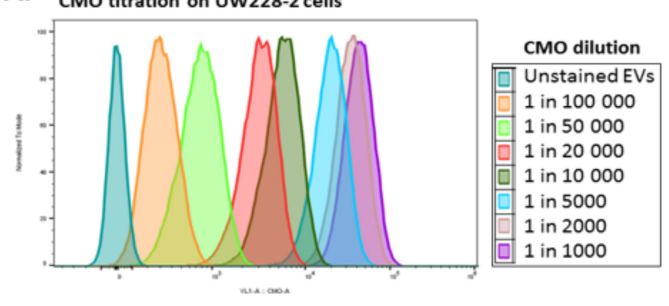

B.
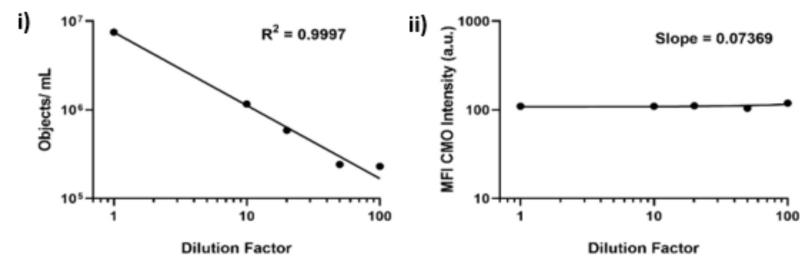

C.
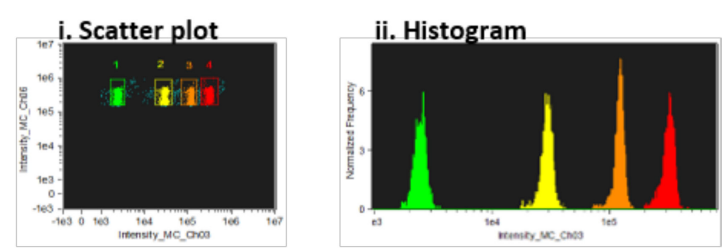

iv. Regression analysis

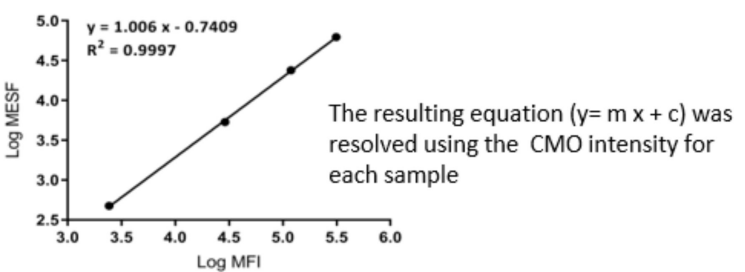

iii Quantibrite PE Bead log MFI and MESF Calculation

\begin{tabular}{ccccc}
\hline & MFI & Log MFI & $\begin{array}{c}\text { MESF * } \\
\text { (PE Mols/ Bead) }\end{array}$ & Log MESF \\
\hline Low & 2430.8 & 3.3857 & 474 & 2.6758 \\
Med-Low & 29022.98 & 4.4627 & 5359 & 3.7291 \\
Med-High & 119302.62 & 5.0766 & 23843 & 4.3774 \\
High & 313158.76 & 5.4958 & 62336 & 4.7947 \\
\hline \multicolumn{3}{c}{} & \multicolumn{3}{c}{${ }^{*}$ Manufacturer supplied }
\end{tabular}

v. Solve the equation to assign a MESF value

\begin{tabular}{|c|c|c|c|c|c|c|c|}
\hline & & Min MFI & y (LOG10 MFI) & m & $x$ & c & MESF \\
\hline \multirow{4}{*}{ 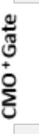 } & 1 & 272.93 & 2.436 & 0.9997 & 1.6957 & 0.7409 & 49.620 \\
\hline & 2 & 273.17 & 2.436 & 0.9997 & 1.6960 & 0.7409 & 49.664 \\
\hline & 3 & 273.27 & 2.437 & 0.9997 & 1.6962 & 0.7409 & 49.682 \\
\hline & 4 & 273.5 & 2.437 & 0.9997 & 1.6966 & 0.7409 & 49.724 \\
\hline & & Max MFI & y (LOG10 MFI) & m & $x$ & c & MESF \\
\hline & US & 271 & 2.433 & 0.9997 & 1.693 & 0.7409 & 49.269 \\
\hline
\end{tabular}

Figure 2. Cont. 
D. i) Loss of $\mathrm{CMO}$ labelling following the addition of Triton-X confirms EV membrane labelling

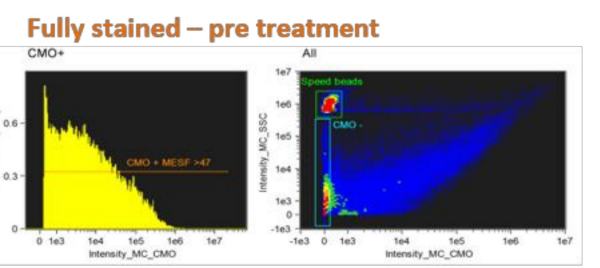

Fully stained - post Triton-X treatment

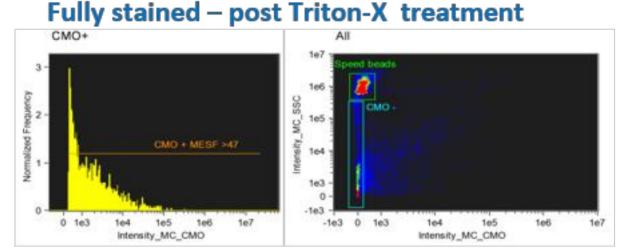

ii) Overlay showing $\mathrm{CMO}$ intensity of unstained and fully stained EVs pre and post Triton-X treatment

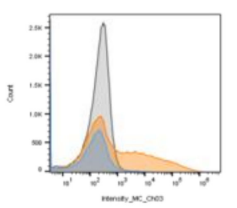

Unstained

Fully stained - pre treatment

Fully stained - post Triton-X treatment

Figure 2. Cell Mask Orange can label EVs to enable standardisation across experiments. (A) Cell Mask Orange (CMO) labelling was optimised by titration using parent cells. Serial dilutions from 1 in 1000-100,000 were used to label parent UW228-2 cells and analysed by flow cytometry. A clear relationship between $\mathrm{CMO}$ concentration and fluorescence intensity is shown. (B) ISX: Objects/mL reduce with increased sample dilution (i) whilst CMO intensity remains constant (ii). Sample dilution was used to validate EV staining protocol. Using a CMO dilution of 1 in $2000(2.5 \mu \mathrm{g} / \mathrm{mL})$, the Imagestream acquired fewer $\mathrm{CMO}$ positive objects per $\mathrm{ml}$ with increasing dilution of $\mathrm{CMO}$ labelled EVs (i), whilst the CMO fluorescence intensity in channel 03 was maintained (ii). (C) Quantibrite PE Beads were run at the time of each experiment and used to assign molecules of equivalent soluble fluorochrome (MESF) values to the CMO intensity of labelled EVs. (Representative example from a single experiment shown) Quantibrite PE beads were acquired at the time of each experiment to assign MESF value. Quantibrite PE beads were separated on a bivariate plot of intensity against side scatter (i). The fluorescence intensity of each bead set was gated on the histogram (ii) and assigned a MESF value according to the number of PE molecules per bead as provided by the manufacturer (iii). Log MFI against Log MESF provided a standard curve for each acquisition (iv). Regression analysis was used to extrapolate the equivalent MESF value for CMO intensity for unstained and labelled samples (v) which enabled lower threshold for CMO intensity to be set to distinguish between unstained and CMO labelled EVs. Representative experiment shown. MESF 50 was subsequently used to set the lower $\mathrm{CMO}+$ threshold to standardise across experiments. (D) CMO labelling was diminished following the addition of detergent. $\mathrm{CMO}$ positive (CMO+) EVs could be distinguished from unstained EVs by ISX (i). Treatment of the same sample with Triton-X 100 diminished the CMO labelling (ii). Exported .fcs files from the same acquisition were overlaid using FlowJo 10.6 (Ashland). The fully stained sample (orange) shows increased fluorescence intensity in channel 3. The same sample post Triton-X treatment (blue) showed a reduction in fluorescence intensity to a similar level of the unstained sample (grey).

\subsection{Large EVs Size Distribution and Quantity was Assessed Using Tunable Resistance Pulse Sensing (TRPS)}

At the time of each preparation, an aliquot of freshly isolated EVs was assessed using the qNano GOLD particle counter to ascertain EV size distribution (diameter) in terms of percentage population $(\%)$ and concentration (particles/mL) prior to labelling for ISX analysis. Using a series of 3 overlapping Nanopores (Figure 3A(i)), each with an optimal size range which spans a total size of approximately $275 \mathrm{~nm}$ to $5.7 \mu \mathrm{m}$, we assessed the size range of particles in the EV preparations. Representative profiles of diameter against percentage population are shown for a single sample using NP600, NP1000, and NP2000 Nanopores (Figure 3A(ii-iv)). By selecting overlapping Nanopore sizes, the same calibrator beads $(1000 \mathrm{~nm})$ could be used and therefore it was possible to overlay the resulting profiles to visualise the total large EV population within each sample (Figure $3 \mathrm{~A}(\mathrm{v})$ ). As expected, the EVs present in each biological replicate were heterogeneous in size. However, the size range of EVs across biological replicates was consistent $(250 \mathrm{~nm}$ to $6 \mu \mathrm{m})$; with some variation in the median diameter per sample. Size is presented with a bin of $100(\mathrm{~nm})$ and in each case the most prevalent large EVs were detected using 
the NP600. The median size across the replicates using the NP600 for comparison were 250-350 nm $5.15 \times 10^{7} ; 450-550 \mathrm{~nm} 1.7 \times 10^{7}$, and $250-350 \mathrm{~nm} 2.8 \times 10^{8}$. The starting volume of EV-containing media used per replicate was $100 \mathrm{~mL}$. The resulting pellet was re-suspended in a total of $700 \mu \mathrm{L}$ PBS (prior to labelling). As an example, applying this dilution factor (142.86) to the first replicate equates to $7.4 \times 10^{9}$ EVs specifically of the $250-350 \mathrm{~nm}$ size range that were released by $7.9 \times 10^{6} \mathrm{UW} 228-2$ cells (NB cell count at harvest) in $24 \mathrm{~h}$ into $100 \mathrm{~mL}$ serum-free media. It should be noted that due to the number of washes and centrifugation steps during the isolation, this can only be considered as an illustration of the quantity of EVs produced. The total larger EVs as counted using the NP2000 $(935 \mathrm{~nm}-5.7 \mu \mathrm{m})$ were less common but are nevertheless abundantly present in concentrations of $2.3 \times 10^{8}, 6.9 \times 10^{8}$ and $2.1 \times 10^{9}$ in the $100 \mathrm{~mL}$ harvested media from this particular cell line. The total particle count for each Nanopore size, across 3 biological replicates is shown in Table 1. It should be noted that the counts shown are not cumulative as they represent the same sample analysed across 3 Nanopore sizes.

A. TRPS Analysis using the qNano instrument determines large EV quantity and size range

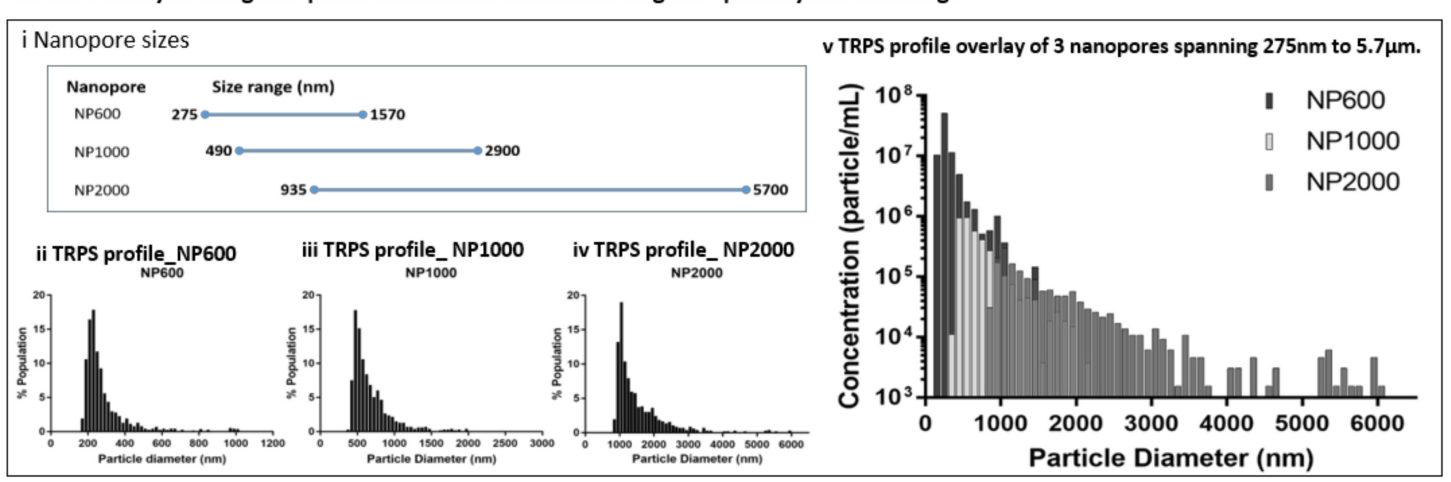

B. Size calibration beads were used to determine the most appropriate diameter mask to use for ISX analysis

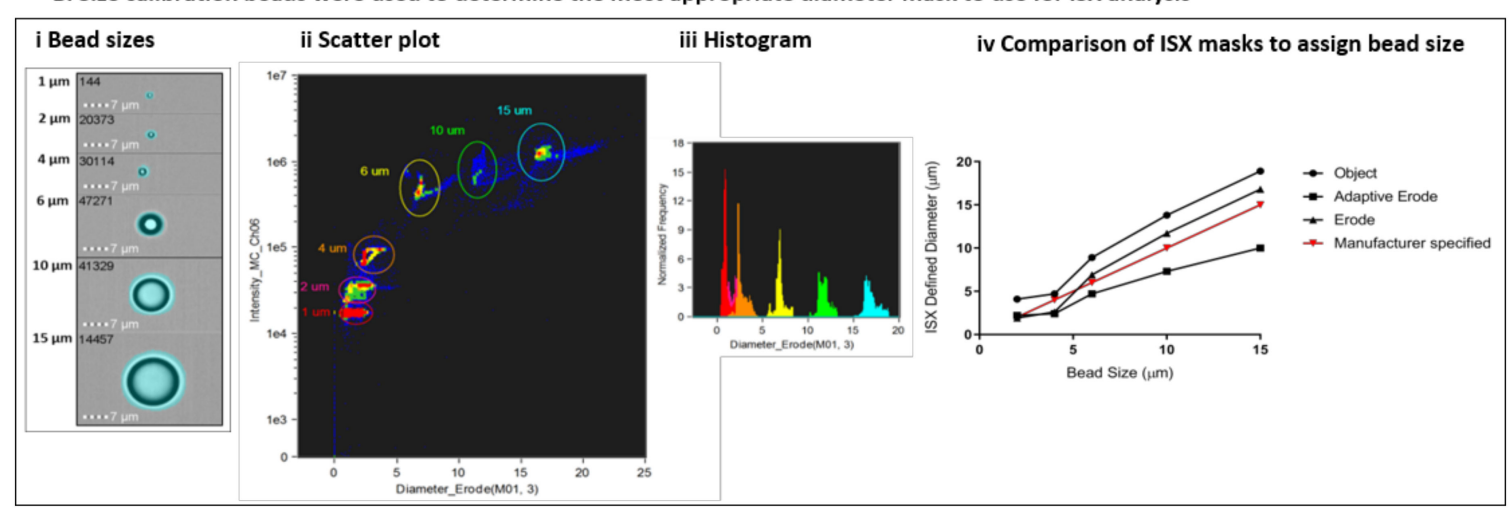

Figure 3. EV size and quantity was assessed by Tunable Resistance Pulse Sensing (TRPS) using the qNANO instrument (iZON Science) and compared to diameter masks in the ISX IDEAS software. (A) Three Nanopores of over-lapping size ranges were used to determine the particle diameter and concentration in each EV preparation (i). Size profiles were established for each individual Nanopore (ii NP600; iii NP1000; iv NP2000). The overlaid profiles of diameter by concentration provided by the 3 Nanopores show a size range of $250 \mathrm{~nm}$ and $6 \mu \mathrm{m}$ against a common calibrator bead of $1000 \mathrm{~nm}$ (representative sample shown) (v). (B) Calibration beads of known size were used to determine the most accurate diameter mask. The masks available within the ISX IDEAS software for diameter analysis were compared. The 3 which assigned the most accurate sizes according to manufacturer specified beads sizes were the Object, Erode and Adaptive Erode masks. These were compared across bead size by applying the mask (ii) visualising a scatter plot (iii) and histogram for each mask type. For the size range within our EV preparations, we found the Erode mask to be the most accurate (iv). 
Table 1. Total particle count using different Nanopore sizes (qNANO) in biological replicates.

\begin{tabular}{cccc}
\hline- & NP600 & NP1000 & NP2000 \\
\hline 1 & $8.4 \times 10^{8}$ & $3.8 \times 10^{6}$ & $1.6 \times 10^{6}$ \\
\hline 2 & $4.9 \times 10^{7}$ & $1.4 \times 10^{7}$ & $4.8 \times 10^{6}$ \\
\hline 3 & $5.0 \times 10^{8}$ & $4.4 \times 10^{7}$ & $1.5 \times 10^{7}$ \\
\hline
\end{tabular}

Size calibration beads were used to determine the most appropriate diameter mask to use for ISX analysis of EV diameter. The bead sizes provided by the manufacturer were 1, 2, 4, 6, 10, and $15 \mu \mathrm{m}$. We used the feature tool in IDEAS to apply a range of different diameter masks to the bright field image of the beads (Figure 3B(i)). We selected bright field for this using non-labelled beads because the Quantibrite beads showed over exaggeration of EV size, possibly due to saturation and flare of fluorescence. The beads were visualised on bivariate plots of diameter versus side scatter (channel 06). Density plots (Figure 3B(ii)) allowed the bead populations to be gated individually and subsequent histograms (Figure 3B(iii)) to be viewed. We compared the diameters for each bead population assigned using the Object, Adaptive Erode and Erode Masks against the manufacturer specified size (Figure $3 \mathrm{~B}(\mathrm{iv})$ ) and found the Erode mask on the bright field image, using 3 pixel reduction to be the most comparable.

\subsection{Fluorescence Membrane Labelling Can Be Used to Distinguish EVs from Background and Speed Beads}

We devised an analysis template within IDEAS which comprised a hierarchical gating strategy aimed at characterising heterogeneous large EVs (Figure 4A). All buffers were filtered using a $0.2 \mu \mathrm{m}$ filter and samples were acquired for $5 \mathrm{~min}$ to avoid recording different amounts of background per acquisition. Speed beads were included. The speed beads and $\mathrm{CMO}^{-}$events were defined using density plots of channel $03(\mathrm{CMO}$ ) versus channel 06 (side scatter) intensity (Gate i). Running acquisition buffer only (left side) and unstained EVs (centre) showed the instrument detected a high level of background with low to medium side scatter. Labelling with the cell membrane dye (CMO) therefore helped to distinguish between the low CMO intensity/low side scatter EVs and background detected in both the acquisition buffer and unstained sample (right side). Applying a gate to capture low Raw Max Pixel events (channel 06-SSC: Gate ii) eliminated those events with saturated side scatter, including the speed beads. Outliers in the side scatter versus CMO intensity plots were individually inspected and found to be dual events comprising a speed bead $\left(\mathrm{CMO}^{-}\right.$, high SSC) and an EV (CMO+, low SSC) which occupied the flow chamber at the same time. This resulted in aberrant events with both high $\mathrm{CMO}$ intensity and high SSC and was therefore excluded from further analysis.

Events classed as $\mathrm{CMO}+$ at this point were included in the initial $\mathrm{CMO}+$ gate (Gate iii). Within the same acquisition, we ran the Quantibrite PE beads which enabled a minimum threshold for $\mathrm{CMO}+$ events to be calculated and converted to MESF units, as described. The lower MESF cut off for this experiment was defined at $>50$ and a further gate on the CMO intensity histogram (Gate iv) captured all $\mathrm{CMO}+$ events with an MESF $>50$ for downstream analysis. The proportion of $\mathrm{CMO}+$ events as a percentage of all events acquired over the $5 \mathrm{~min}$ period varied across 10 replicates (mean $18.73 \% \pm 1.2 \mathrm{SEM}$ ) CMO+ MESF > 50; Figure 4B). A final gate was included to eliminate outliers which were either high CMO intensity but appeared on the bright field image to be membrane fragments (Gate v) or were not assigned a diameter due to lack of bright field image focus. This stringent gating strategy was designed to eliminate any events which could not be further analysed for EV marker expression or diameter. 


\section{A ISX Gating Hierarchy}

All samples acquired for 5 minutes

\begin{tabular}{|c|}
\hline $\begin{array}{c}\text { All } \\
\text { Scatter plot: CMO intensity v SSC }\end{array}$ \\
\hline $\begin{array}{l}\text { Gate i: Speed beads and } \\
\text { background/CMO }\end{array}$ \\
\hline $\begin{array}{c}\text { All } \\
\text { Histogram: Raw Max Pixel_MC_Ch06 }\end{array}$ \\
\hline Gate ii: Non-saturated events \\
\hline$\downarrow$ \\
\hline $\begin{array}{l}\text { Non-saturated events } \\
\text { Scatter plot: CMO Intensity v SSC }\end{array}$ \\
\hline $\begin{array}{l}\text { Gate iii: } \mathrm{CMO}^{+} \text {using co-ordinates to set } \\
\text { lower level from unstained EVs }\end{array}$ \\
\hline$\downarrow$ \\
\hline $\begin{array}{l}\mathrm{CMO}^{+} \\
\text {Histogram: } \mathrm{CMO}^{\text {Intensity }}\end{array}$ \\
\hline
\end{tabular}

Gate iv: Anchor lower threshold to CMO intensity equating to MESF 50 *

* Calculated per experiment (ISX run) using Quantibrite PE Beads (Channel 03)

\begin{tabular}{|c|}
\hline $\begin{array}{c}\text { Apply diameter mask to bright field } \\
\text { images }\end{array}$ \\
\hline $\begin{array}{c}\text { CMO + MESF }>50 \\
\text { Scatter plot: CMO intensity } v \text { Diameter }\end{array}$ \\
\hline Gate v: Refined gate to eliminate \\
outliers \\
$\downarrow$ \\
\hline $\begin{array}{c}\text { Refined gate } \\
\text { Scatter plot: BV421 v AF647 } \\
\text { Apply positive gates defined using } \\
\text { FMO controls }\end{array}$ \\
\hline
\end{tabular}

Save template and apply to all fully stained samples per experiment.

All experiments were performed a minimum of 3 times
Gate i: Speed beads and background/ $/ \mathrm{CMO}^{-}$

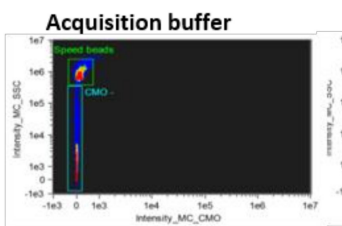

Unstained EVs

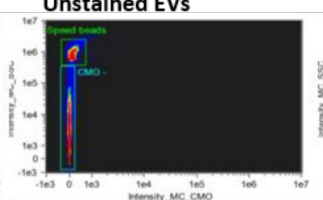

CMO Labelled EVs

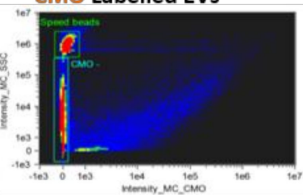

Gate ii: Non-saturated events

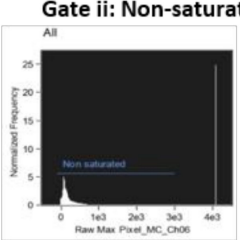

Gate iv: $\mathrm{CMO}^{+} \mathrm{MESF}>\mathbf{5 0}$

cmo+

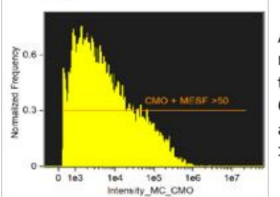

An MESF value was assigned to the minimum $\mathrm{CMO}$ intensity value from the $\mathrm{CMO}^{+}$gate according to the Quantibrite PE Beads in each acquisition and a standardised MESF 50 designated a CMO+ event 50 designated $\mathrm{a} \mathrm{CMO}^{+}$event

$\mathrm{CMO}+$ Gate lower threshold set above the CMO - gate from the unstained EV sample for each experiment

B \% positive $\mathrm{CMO}^{+}$events (MESF >50)

Gate v: Refined Gate to eliminate outliers CMO+ MESF 250

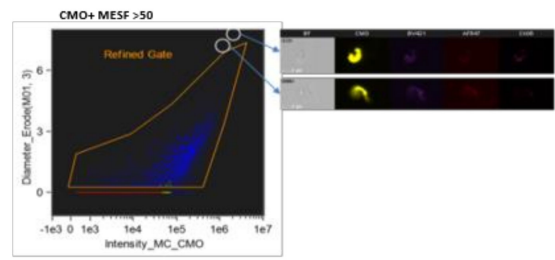

C Compensation matrices

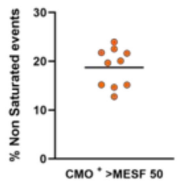

D Fluorescence minus one (FMOs) controls were used to set positive gates i) FMO AF647

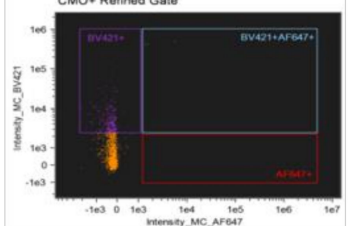
ii) FMO BV421

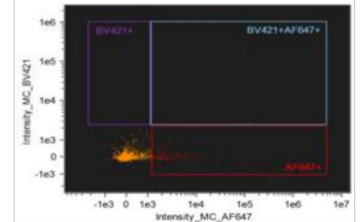

E UW228-2 cells show uniform membrane labelling using CMO at 1 in 2000 and express CD63, CD9 and LAMP1
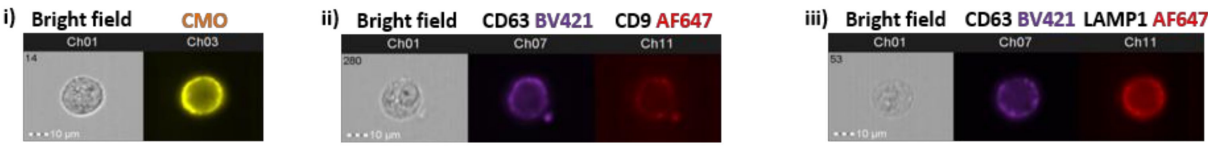

Figure 4. Optimised acquisition by Imaging flow cytometry and florescence membrane labelling can distinguish large extracellular vesicles from background and enable multiplex labelling. (A) Hierarchical gating can confidently refine the EV population for characterisation. Samples were acquired for $5 \mathrm{~min}$ using the ISX INSPIRE software and all events visualised using bivariate plots for fluorescence intensity in channel 03 (CMO) and channel 06 (side scatter). Initial gates for speed beads and $\mathrm{CMO}^{-}$events were set using the Acquisition buffer only and unstained EV samples included in each run (Gate i). These gates were applied to the labelled samples. Saturated events were excluded from the analysis by gating on the histogram plot: Raw Max Pixel for Channel 06 (Gate ii) thus removing the speed beads and very high side scatter events. An initial $\mathrm{CMO}+$ gate was placed (Gate iii). A lower fluorescence threshold of MESF > 50 in channel $3(\mathrm{CMO})$ was set in each experiment using the regression analysis of Quantibrite PE Beads as described. The $\mathrm{CMO}+$ gate was anchored using co-ordinates for the equivalent fluorescence intensity and a further CMO + MESF > 50 gate applied to the histogram (Gate iv). Finally, a refined gate was used to eliminate outliers (Gate v). The analysis template was set for each experiment 
and applied to all samples. (B) Percentage positive $\mathrm{CMO}+$ events (MESF $>50$ ). The percentage of $\mathrm{CMO}+$ events (MESF > 50) is represented by an orange dot for 10 replicate experiments. Black bar represents mean $18.73 \%$ ( $\pm 1.2 \mathrm{SEM})$. (C) A compensation matrix was applied to all samples. The compensation wizard was used to create a matrix which was applied to all samples. (D) Single, double, and triple labelling protocols were used for compensation and accurate gating. Dual labelled EVs provided Fluorescence minus one (FMO) controls for (i) AF647; EVs labelled with CMO and CD63 BV421) or (ii) BV421; (EVs labelled with CMO and LAMP1 AF647) and used to set positive gates. (E) UW228-2 cells were used to optimise multiplex labelling. Cell mask orange $(\mathrm{CMO})$ provides a general membrane label (i) and could be used to co-label with EV markers CD63 BV421 and CD9 AF647 (ii) or CD63 BV421 and LAMP1 AF647 (iii). Representative gallery images from IDEAS software are shown.

We tested a number of approaches to apply compensation to our data in consultation with the manufacturer's specialists. Initially, we attempted to construct a matrix using single stained EVs; however, the single pixel fluorescence was insufficient for the in-built compensation Wizard to assign a matrix. Next, we tried single stained parent cells, but the compensation matrix formulated by the wizard resulted in over-compensation and negative fluorescence intensity events. Over-compensation was thought to be due to the imbalance between the strong fluorescence signal resulting from cell mask orange, a membrane marker which indiscriminately labels lipids, and relatively weak fluorescence signal from target specific antibodies. These experiments were originally performed using a FITC conjugated CD63 antibody, however we found the level of adjustment required between channel 02 (FITC) and channel 03 (CMO) contributed to the negative populations. We re-optimised using the same CD63 antibody clone (HSC6) conjugated to BV421 (channel 07) which is spectrally more distinct from CMO. Finally, we found that using commercially available compensation beads labelled with our antibodies and acquired in the channels used for our study, in conjunction with assisted manual adjustment, provided the most reproducible compensation matrix.

We combined CMO with BV421 and AF647 and Fluorescence Minus One (FMO) controls for each fluorophore were used to set positive gates (Figure 4D). The parent UW228-2 cells were screened for expression of the EV markers chosen for this study: CD63, CD9, and LAMP1 as defined in the latest MISEV guidelines [1]. Multiplex labelling was performed using the following combinations: CMO with CD63 BV421 and CD9 AF647; or CMO with CD63 BV421 and LAMP1 AF647.

\subsection{Multiplex Labelling Reveals Heterogeneity in EV Marker Expression}

The proportion of $\mathrm{CMO}+$ only events varied across the 10 replicates (Figure 5A). The mean percentage positive $\mathrm{CMO}+(\mathrm{MESF}>50)$ events $(63.3 \% \pm 4.6 \mathrm{SEM})$ which did not demonstrate expression of any EV markers included in this study were designated CMO only. Considering the single EV markers, the proportion of EVs positive for each EV marker varied CD63 19.0\%, ( \pm 2.2 SEM), CD9 mean $10.3 \%$ ( \pm 0.5 SEM); LAMP1 47.6\% ( \pm 3.8 SEM). These data shown LAMP1 to be the most prevalent EV marker screened in this study.

\section{A Within the $\mathrm{CMO}^{+}$(MESF >50) gate: The percentage positive $\mathrm{CMO}$ only events which did not express the EV markers screened in this study varied across replicates. The proportion of $\mathrm{CMO}^{+}$events expressing a single $\mathrm{EV}$ marker also varied across replicate experiments with LAMP1 being the most prevalent.}

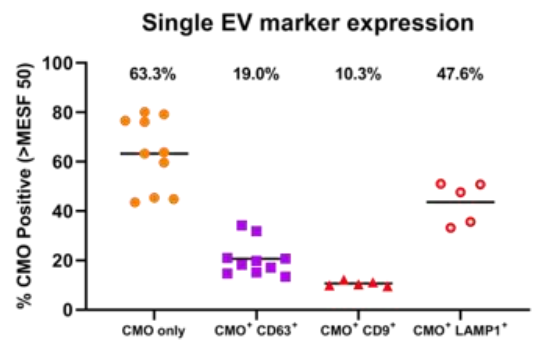

Figure 5. Cont. 


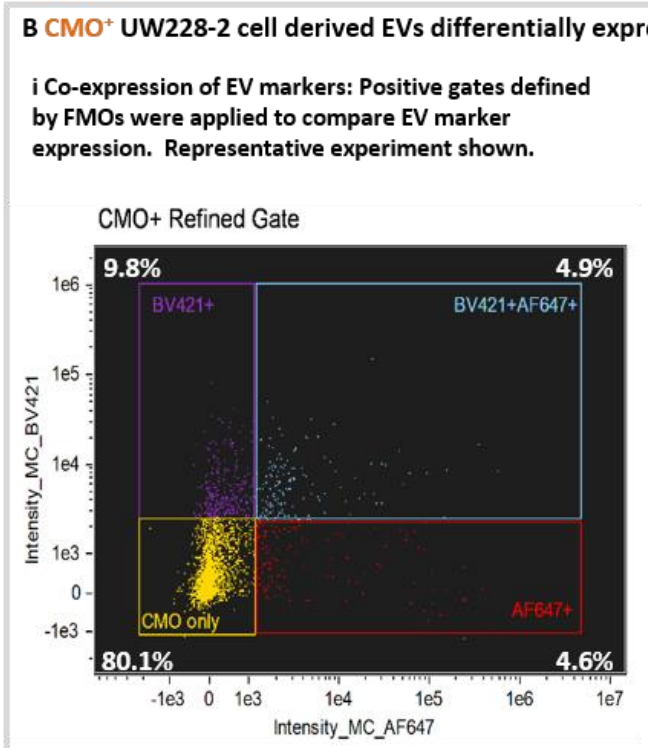

ii ISX Gallery images show individual EVs from each region can be inspected.

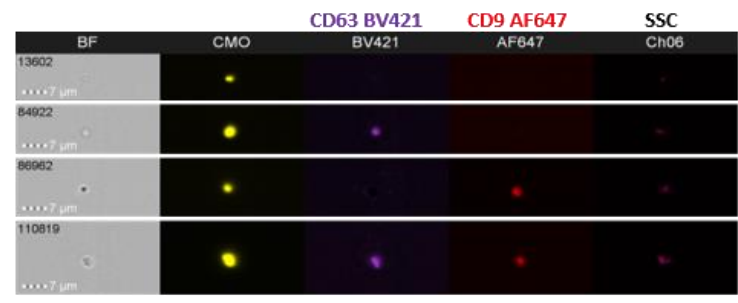

iii \% CMO positive (MESF >50) EVs which co-express EV markers. Black bar indicates mean \% positive across 5 experiments.

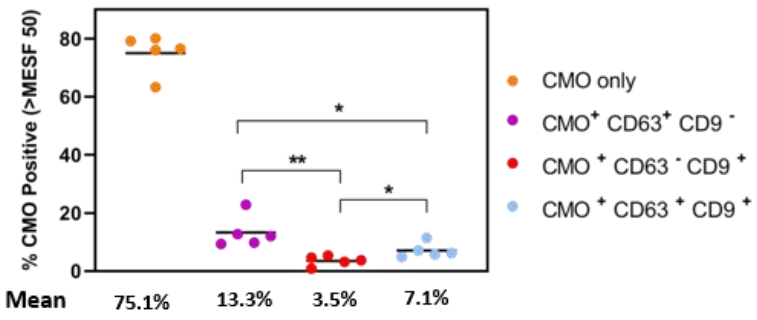

C CMO+ UW228-2 cell derived EVs differentially express CD63 BV421 and LAMP1 AF647.

i Co-expression of EV markers: Positive gates defined by FMOs were applied to compare EV marker expression. Representative experiment shown.

ii ISX Gallery images show individual EVs from each region can be inspected.
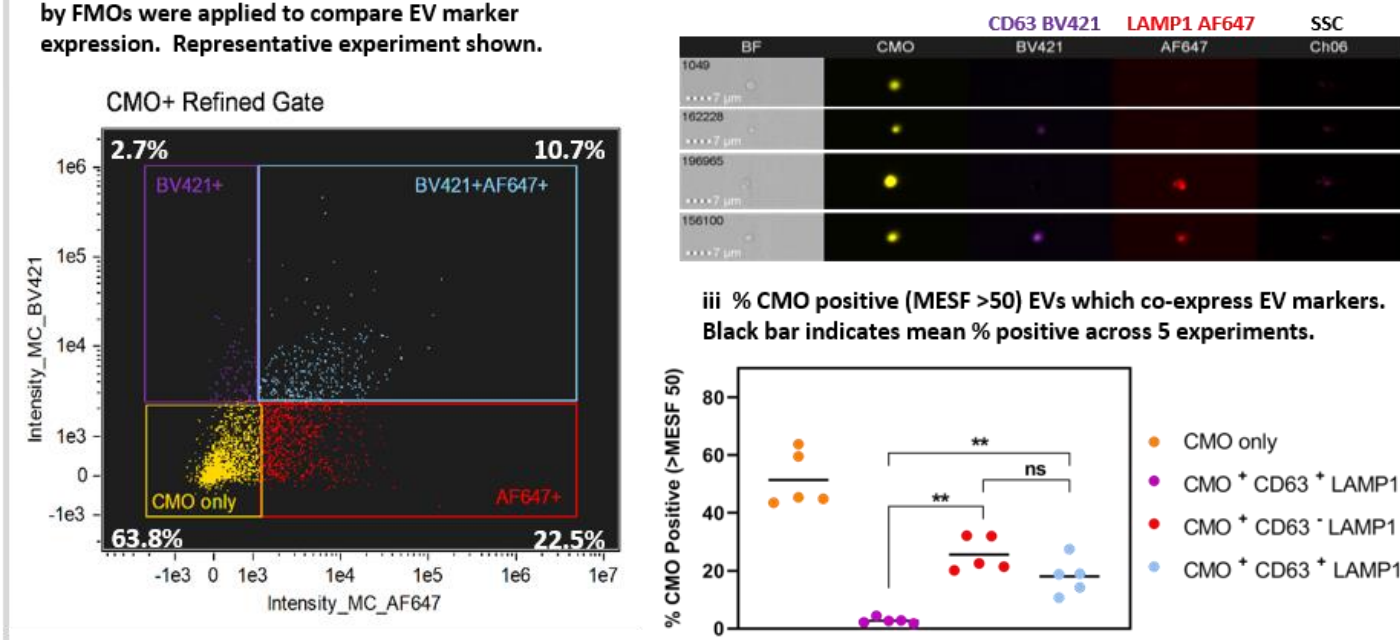

iii \% CMO positive (MESF >50) EVs which co-express EV markers. Black bar indicates mean \% positive across 5 experiments.

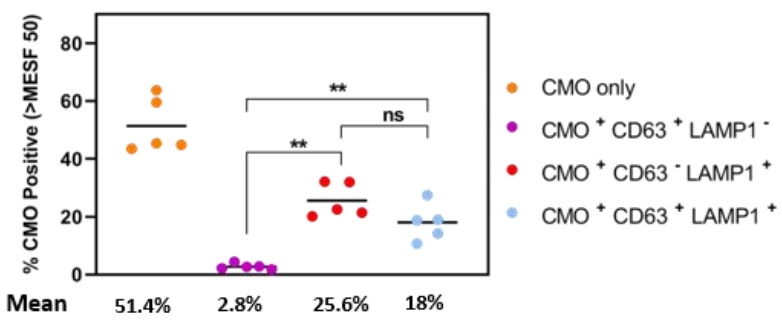

Figure 5. Isolated large EVs can be triple-labelled and individual events can be scrutinised post-acquisition. (A) The proportion of $\mathrm{CMO}+$ events expressing a single EV marker varied across replicates. The percentage positive events within the $\mathrm{CMO}+\mathrm{MESF}>50$ gate which expressed either no EV marker (CMO only), CD63, CD9, or LAMP1 varied across replicates. Black bar represents the mean across 10 (CMO or CD63) or 5 (CD9 or LAMP1) replicates. CMO only 63.3\% ( \pm 4.6 SEM), CD63 only $19 \%$ ( \pm 2.2 SEM), CD9 ( \pm 0.5 SEM) only $10.3 \%$ and LAMP1 only $47.6 \%$ ( \pm 3.8 SEM). (B) UW228-2 cell derived EVs differentially express EV markers CD63 and CD9, or both. EVs were labelled with CMO, CD63 BV421 and CD9 AF647. CMO ${ }^{+}$events (MESF > 50) were analysed for co-expression with CD63, CD9 or both. Quadrant gating of bivariate plots for fluorescence intensity in channel 07 (BV421) and channel 11 (AF647) demonstrated single labelled $\left(\mathrm{CMO}^{+}\right.$only), dual and triple labelled EV populations (i). Percentage positive in each quadrant is shown. Representative experiment. Gallery images display examples of individual events from each of the 4 quadrants (ii). Bright Field (BF) and side scatter (SSC) channels are shown alongside CMO, CD63 BV421 and CD9 AF647. Of the CMO+ MESF >50: 75.1\% $( \pm 3.0 \mathrm{SEM})$ were $\mathrm{CMO}^{+}$only; $13.3 \%( \pm 2.4 \mathrm{SEM})$ expressed CD63 BV421 and 3.5\% ( $\left.\pm 0.8 \mathrm{SEM}\right)$ 
expressed CD9 AF647. 7.1\% ( \pm 1.1 SEM) of the $\mathrm{CMO}^{+}$events expressed both CD63 and CD9. (C) UW228-2 cell derived EVs differentially express EV markers CD63 and LAMP1, or both. EVs were labelled with CMO, CD63 BV421 and LAMP1 AF647. CMO ${ }^{+}$events were analysed for co-expression with CD63, LAMP1 or both. Quadrant gating of bivariate plots for fluorescence intensity in channel 07 (BV421) and channel 11 (AF647) demonstrated single labelled ( $\mathrm{CMO}^{+}$only), dual and triple labelled EV populations (i). Percentage positive in each quadrant is shown. Representative experiment. Gallery images display examples of individual events from each of the 4 quadrants (ii). Bright Field (BF) and side scatter (SSC) channels are shown alongside CMO, CD63 BV421 and LAMP1 AF647. Of the CMO+ MESF > 50: 54.1\% ( $\pm 4.3 \mathrm{SEM})$ were $\mathrm{CMO}^{+}$only; $2.8 \%$ ( $\left.\pm 0.4 \mathrm{SEM}\right)$ expressed CD63 BV421 and $25.6 \%$ ( \pm 2.7 SEM) expressed LAMP1 AF647 whilst 18.0\% $( \pm 2.8 \mathrm{SEM})$ of the $\mathrm{CMO}^{+}$events expressed both CD63 and LAMP1. ( $p=0.0159$ or $\left.0.0317 ;{ }^{* *} p=0.0079\right)$. Mann Whitney U test.

Differential expression and co-expression of EV markers was examined between five replicate experiments. Representative bivariate plots of AF647 intensity against BV421 intensity (Figure 5B(i) and Figure 5C(i) for CD63/CD9 and CD63/LAMP1, respectively), and representative galleries (Figure 5B(ii) and Figure 5C(ii)) of EVs displaying each labelling combination taken from the quadrant plots are shown. The proportion of EVs which co-express EV markers within each gate for the five replicates is shown in Figure 5B(iii) and Figure 5C(iii). Where the EVs were labelled for CMO, CD63 BV421, and CD9 AF647 (Figure 5B(iii)): 75.1\% ( \pm 3.0 SEM; yellow) were CMO only, 13.3\% ( \pm 2.4 SEM; purple) were $\mathrm{CMO}^{+} \mathrm{CD}^{+} 3^{+} \mathrm{CD}{ }^{-} ; 3.5 \%$ ( $\pm 0.8 \mathrm{SEM}$; red) were $\mathrm{CMO}{ }^{+} \mathrm{CD}^{-} 3^{-} \mathrm{CD}^{+}{ }^{+}$and $7.1 \%( \pm 1.1 \mathrm{SEM}$; blue) were $\mathrm{CMO}+\mathrm{CD} 63{ }^{+} \mathrm{CD} 9{ }^{+}$. There were significantly fewer $\mathrm{CD} 63-\mathrm{CD} 9{ }^{+}$EVs compared with $\mathrm{CD} 3^{+}(p=0.0079)$ only or $\mathrm{CD} 63^{+} \mathrm{CD} 9{ }^{+} \mathrm{EVs}(p=0.0159)$. Similarly, there were fewer EV co-expressing $\mathrm{CD} 63$ and $\mathrm{CD} 9$ compared with $\mathrm{CD}^{+}{ }^{+}$alone $(p=0.0317)$. (Mann Whitney $\mathrm{U}$ test). Considering the EVs labelled with CMO, CD63 BV421 and LAMP1 AF647 (Figure 5C(iii)). 51.4\% (+/- 4.3 SEM; yellow) were $\mathrm{CMO}+$ only, a comparatively low $2.8 \%$ ( $\pm 0.4 \mathrm{SEM}$; purple) were $\mathrm{CMO}^{+} \mathrm{CD} 3^{+}{ }^{+} \mathrm{LAMP}^{-}{ }^{-}$; whilst $25.6 \%$ ( $\pm 2.7 \mathrm{SEM}$; red) were $\mathrm{CMO}^{+}{ }^{+} \mathrm{CD} 3^{-}$LAMP1 $^{+}$and $18 \%$ ( $\pm 2.8 \mathrm{SEM}$; blue) co-expressed both CD63 and LAMP1. There were significantly more EVs which expressed LAMP1 compared with CD63 alone $(p=0.0079)$ and more expressing both CD63 and LAMP1 compared with CD63 alone $(p=0.0079)$.

\subsection{ISX Allow Correlative Analyses of Diameter with EV Marker Expression for Phenotyping}

We applied the Diameter Mask; Erode $(M 01,3)$ in IDEAS software to all fully labelled replicates using the Batch analysis function and compared the range of diameters present within the $\mathrm{CMO}+$ MESF $>50$ gate. Figure 6A shows single event diameters within the 10 replicates. This median diameter (indicated with the black line) varied significantly across the replicates (922-1129 nm: Kruskal-Wallis; $p>0.0001$ ). However, these data represent EVs isolated as 3 biological replicates and when each replicate is analysed using ANOVA there was no significant difference between the median diameters of each EV preparation. There was a clear correlation between EV diameter and CMO intensity as shown in Figure 6B. Regression analysis was performed on the individual exported feature values from each replicate ( 2 representative plots are shown from different biological replicates) $(p<0.0001)$.
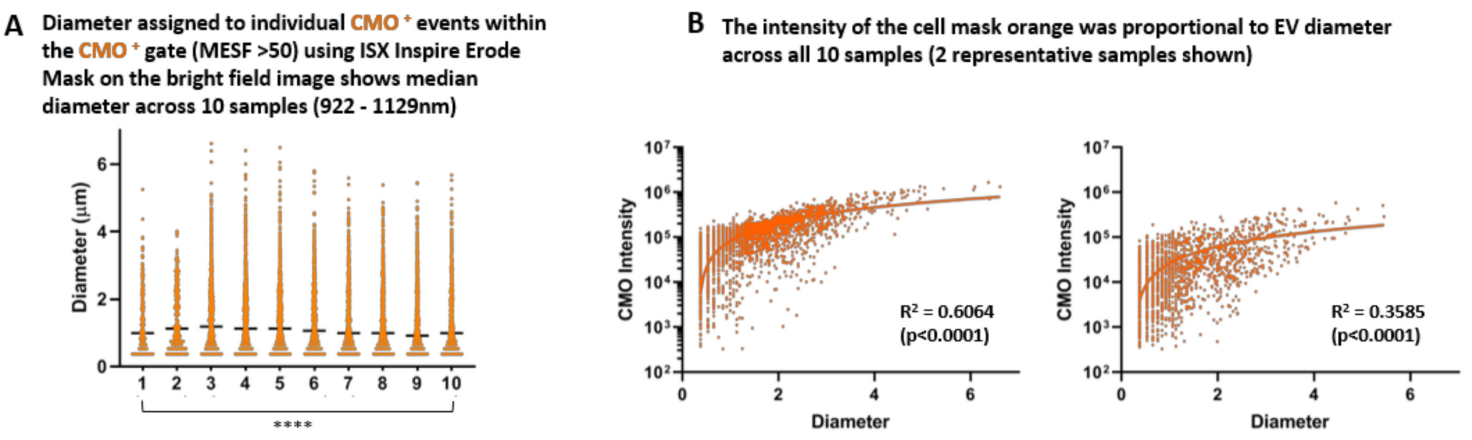

Figure 6. Cont. 
C Large EVs are heterogeneous in diameter and EV marker expression

$\mathrm{CMO}+\mathrm{EV}$ markers CD63 and CD9

CMO + EV markers CD63 and LAMP1

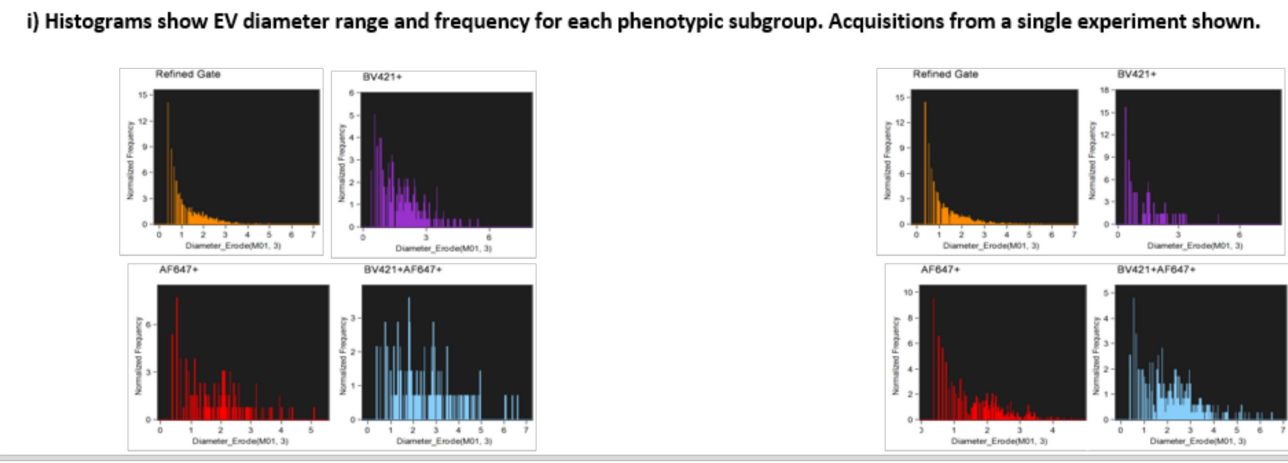

ii) Large EVs are heterogeneous in diameter within each phenotypic subgroup. Representative spread of EV diameters from a single replicate is shown. Black bar shows median diameter for each phenotype.
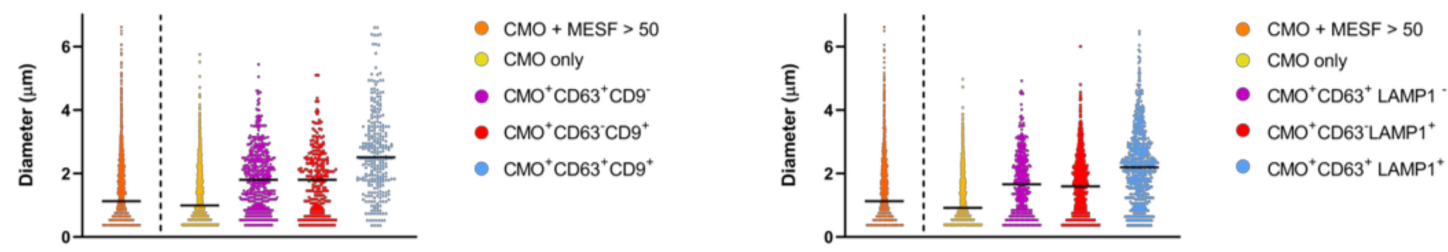

iii) Large EVs co-expressing $2 \mathrm{EV}$ markers are significantly bigger than those not expressing any EV markers included in this study
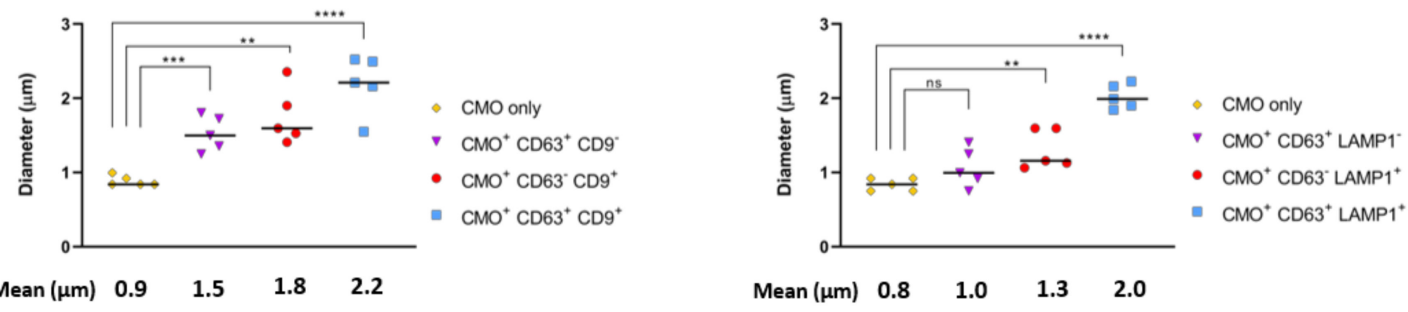

Figure 6. The ISX can accurately assign diameter to large EVs and facilitate individual event analyses to phenotype heterogeneous EV populations. (A) The median diameter of $\mathrm{CMO}^{+}$EVs from UW228-2 cells was comparable across 10 samples. The erode mask was applied to the bright field images of those EVs which were included in the $\mathrm{CMO}^{+}>\mathrm{MESF} 50$ gate. The diameter of individual events within this gate was exported from IDEAS into PRISM for analysis. This figure shows a median diameter (represented by the black bar) of 922-1129 $\mathrm{nm}$ across the 10 samples and visualises the broad size range within each sample. ${ }^{* * * *} p>0.0001$ Kruskal-Wallis test). (B) The fluorescence intensity of the CMO was proportional to EV diameter. Bivariate plots showing CMO intensity against diameter consistently demonstrates a proportional relationship between EV size and intensity of the membrane label across samples run in triplicate experiments. 2 representative samples shown. (Regression analysis $p<0.0001$ ). (C) Comparison of EV phenotypes across triplicate experiments. Individual histograms displaying EV diameter for each phenotype demonstrated differential size ranges according to EV marker(s) expression ( $\mathrm{i}$ a and $\mathrm{i}$ b). In each case, those labelled with CMO only and not expressing either CD63, CD9 or LAMP1 have a smaller median diameter compared with EVs expressing CD63 and/or CD9 or LAMP1 (ii a and ii b). In both cases, those EVs co-expressing both CD63 with CD9 (iii a) or CD63 with LAMP1 (iii b) are significantly larger than CMO only. $\left({ }^{* *} p<0.005,{ }^{* * *} p<0.0004,{ }^{* * * *} p<0.0001\right.$ ).

We found that large EVs are heterogeneous in both diameter and EV marker expression. Histogram plots of individual events exported from the quadrant positive gates show the different diameter range and frequency within each phenotypic subgroup (Figure 6C(i)). Representative acquisitions 
from a single replicate are shown. ISX analysis facilitates the comparison of individual EV diameters from within each phenotype (Figure 6C(ii)). A representative plot for all $\mathrm{CMO}^{+}(\mathrm{MESF}>50)$ in a single replicate of each phenotype is shown and the black bars represent the median diameter for each subgroup. Within this typical replicate, the median diameter and range for each subgroup was as follows: All CMO >MESF $501.1 \mu \mathrm{m}(362 \mathrm{~nm}-6.2 \mu \mathrm{m})$, CMO only $1.0 \mu \mathrm{m}(362 \mathrm{~nm}-5.4 \mu \mathrm{m}), \mathrm{CMO}+$ $\mathrm{CD}^{+} 3^{+} \mathrm{CD}^{-} 1.8 \mu \mathrm{m}(362 \mathrm{~nm}-5.1 \mu \mathrm{m}), \mathrm{CMO}+\mathrm{CD}^{-} 3^{-} \mathrm{CD}^{+} 1.8 \mu \mathrm{m}(362 \mathrm{~nm}-4.7 \mu \mathrm{m})$, and CMO+CD63 ${ }^{+}$ $\mathrm{CD}^{+} 2.5 \mu \mathrm{m}(362 \mathrm{~nm}-6.2 \mu \mathrm{m})$. When considering the second phenotype: All CMO >MESF $501.4 \mu \mathrm{m}$ $(362 \mathrm{~nm}-6.2 \mu \mathrm{m}), \mathrm{CMO}$ only $1.1 \mu \mathrm{m}(362 \mathrm{~nm}-4.6 \mu \mathrm{m}), \mathrm{CMO}+\mathrm{CD}^{+}{ }^{+} \mathrm{CD}^{-} 1.7 \mu \mathrm{m}(362 \mathrm{~nm}-4.5 \mu \mathrm{m})$, $\mathrm{CMO}+\mathrm{CD}^{-} 3^{-} \mathrm{CD}^{+} 1.6 \mu \mathrm{m}(362 \mathrm{~nm}-5.6 \mu \mathrm{m})$, and $\mathrm{CMO}+\mathrm{CD}^{+} 3^{+} \mathrm{CD}^{+} 2.2 \mu \mathrm{m}(362 \mathrm{~nm}-6.1 \mu \mathrm{m})$. In each case the median diameter increases in size with accumulating $\mathrm{EV}$ marker expression.

When considering all 5 replicates; we compared the mean diameter for each subgroup (Figure 6C(iii)). For the first group (left panel): CMO only $0.89 \mu \mathrm{m}( \pm 0.03 \mathrm{SEM}), \mathrm{CMO}+\mathrm{CD} 63^{+}$ $\mathrm{CD}^{-} 1.53 \mu \mathrm{m}( \pm 0.1 \mathrm{SEM}), \mathrm{CMO}+\mathrm{CD}^{-} 3^{-} \mathrm{CD}^{+} 1.76 \mu \mathrm{m}( \pm 0.17 \mathrm{SEM})$ and $\mathrm{CMO}+\mathrm{CD}^{+} 3^{+} \mathrm{CD}^{+} 2.19 \mu \mathrm{m}$ $( \pm 0.18$ SEM). Those EVs expressing either CD63 $(p<0.0004)$ or CD9 $(p<0.0010)$ were significantly larger than EV which did not (CMO only). For the second subgroup (right panel) the mean diameters were as follows: CMO only $0.84 \mu \mathrm{m}( \pm 0.04 \mathrm{SEM}), \mathrm{CMO}+\mathrm{CD}^{+} 3^{+} \mathrm{CD}^{-} 1.06 \mu \mathrm{m}( \pm 0.12 \mathrm{SEM}), \mathrm{CMO}+$ $\mathrm{CD} 63^{-} \mathrm{CD}^{+} 1.31 \mu \mathrm{m}( \pm 0.12 \mathrm{SEM})$, and $\mathrm{CMO}+\mathrm{CD}^{+} 3^{+} \mathrm{CD}^{+} 2.02 \mu \mathrm{m}( \pm 0.07 \mathrm{SEM})$. In this group, those EVs expressing CD63 only were not significantly larger than those which did not. However, EVs expressing LAMP1 were larger $(p<0.005)$. In both cases, those $\mathrm{CMO}^{+}$EVs co-expressing $2 \mathrm{EVs}$ markers: either CD63 and CD9, or CD63 and LAMP1 (blue) were significantly larger than those which do not carry the markers screened in this study. (*** $p<0.0001$. Un-paired $t$-test).

\section{Materials and Methods}

\subsection{Cell Line}

UW228-2 cells were kindly provided by DTW Jones (DKFZ, Heidelberg, Germany). Cells were grown in DMEM with L-glutamine (Lonza, Manchester, UK; cat: R8758) supplemented with 10\% FCS (SIGMA, Gillingham, UK; cat: F9665) in Corning $225 \mathrm{~cm}^{2}$ Angled Neck Cell Culture Flask with Vent Cap (Fisher Scientific, Leicestershire, UK; cat: 431082 ) at $37^{\circ} \mathrm{C}$ with $5 \% \mathrm{CO}_{2}$ in normoxia. Cell lines were passaged with $1 \times$ Trypsin-EDTA (Lonza, UK; cat: T3924). All cell lines tested negative for Mycoplasma and all were authenticated in-house (CRUK-Manchester Institute) by examination of a total of 21 loci across the genome using the Powerplex 21 System (Promega, Southampton, UK).

\subsection{Cell Culture}

For each experiment, cells were seeded at $2.5 \times 10^{6}$ cells in $50 \mathrm{~mL}$ DMEM $10 \%$ FBS (complete media) per $225 \mathrm{~cm}^{3}$ tissue culture flask and allowed to adhere overnight. On day 2 media was switched to $50 \mathrm{~mL}$ serum-free DMEM for $24 \mathrm{~h}$ prior to EV isolation and cell preparation. Conditioned media (containing EVs) was removed, and the cells washed $\mathrm{x} 2$ with PBS before trypsinisation using $1 \times$ Trypsin-EDTA (Lonza, Manchester, UK; cat: T3924). Cell counts and viability were checked at the time of EV harvest using the trypan blue exclusion assay (0.4\% Trypan blue solution; SIGMA; Gillingham, UK; cat T8154).

\subsection{Vesicle Isolation}

Large EVs were harvested using a standard operating procedure (SOP) as previously reported (Figure 1F) [15]. For each experiment, EVs were isolated from the serum-free, conditioned media from $2 \times 225 \mathrm{~cm}^{2}$ flasks (pooled; total $100 \mathrm{~mL}$ ). Cell culture supernatant (conditioned media: $\mathrm{CM}$ ) was centrifuged in 2 tubes to remove cells $(300 \times g 5 \mathrm{~min}, \times 2)$ and filtered using a double layered $5 \mu \mathrm{m}$ pore nylon Sieve (Fisher Scientific, Leicestershire, UK; cat 12994257). The supernatant was collected and centrifuged at $2000 \times g$ for $30 \mathrm{~min}$ and prepared for ISX analysis. Centrifugation steps were performed using an Eppendorf 5702 bench top centrifuge with an A-4-38 rotor. All EV preparations 
were performed on the day of analysis and not stored. Experimental procedures for EV isolation have been submitted to the EV-TRACK database (EV TRACK ID: EV190013) [22].

\subsection{Chemicals and Reagents Including Antibodies}

\subsubsection{Apoptosis Assay}

UW228-2 cells were seeded at $1 \times 10^{5 /}$ well into 6 well plates (Corning; Fisher Scientific, Leicestershire, UK; cat CL S3516) and incubated at $37{ }^{\circ} \mathrm{C}$ overnight in DMEM containing $10 \%$ FBS. Triplicate wells were cultured in complete media or switched to serum-free DMEM for $24 \mathrm{~h}$ before screening with Annexin V APC/PI using the Apoptosis detection kit (Biolegend UK, London, UK; cat 640932) according to the manufacturer's instructions. 30,000 events were acquired using the LSR II flow cytometer with lasers for APC (640 nm laser, emission captured at $660 \mathrm{~nm})$ and PI (488 nm laser with emission captured at $575 \mathrm{~nm}$ ). Positive controls were generated to inform accurate gating: cells were treated with $200 \mu \mathrm{M}$ (UW228-2) Etoposide (SIGMA; Gillingham, UK; cat E1383) for $24 \mathrm{~h}$ (Apoptotic cells: Annexin V), or heated at $56^{\circ} \mathrm{C}$ for $10 \mathrm{~min}$ prior to labelling (Dead cells: PI).

\subsubsection{Immunofluorescence Microscopy}

Cells and EVs were immobilised onto CellCarrier 96 well plates (Perkin Elmer; Bucks, UK; Cat 6005550), fixed with 3.7\% paraformaldehyde, permeabilised with $0.2 \%$ Triton X in PBS and probed using anti-human CD63 antibody (Clone: H5C6, Biolegend UK, London, UK; Cat: 353005) directly conjugated to FITC and counterstained for polymerised actin using $0.2 \times$ Alexa Fluor 555 Phalloidin (Fisher Scientific, Leicestershire, UK; cat A34055) and 300 nM DAPI (Biolegend UK, London, UK, 422801). Images were captured using the Perkin Elmer Operetta system (Perkin Elmer; Bucks, UK) at $\times 40$ magnification.

\subsubsection{Transmission Electron Microscopy (TEM)}

EVs were immobilised onto ACLAR (poly-chloro-tri-fluoro-ethylene (PCTFE) film) coated with Corning CellTak (Fisher Scientific, Leicestershire, UK; cat 354240) and fixed with glutaraldehyde in sodium cacodylate buffer ( $\mathrm{pH}$ 7.2) followed by post-fix staining with osmium tetroxide and uranyl acetate (supplied in-house by the FBMH Core Facility). Preparations were dehydrated and embedded in resin to allow serial $60-200 \mu \mathrm{m}$ sections to be taken. Images were captured using a Biotwin Philips TECNAI G2 transmission electron microscope.

\subsubsection{Tunable Resistance Pulse Sensing (TRPS)}

Size and quantity were determined by Tunable Resistance Pulse Sensing (TRPS) using the qNano GOLD instrument (iZON Science, Christchurch, New Zealand) as per manufacturer's instructions. The principles are discussed elsewhere [23]. We analysed an aliquot of isolated EVs alongside downstream analyses using overlapping sizes of Nanopores (NP600, NP1000, and NP2000) to provide a full picture of EV size distribution and quantity.

\subsubsection{Cell Mask Orange Labelling}

$100 \mathrm{~mL}(2 \times 50 \mathrm{~mL}) \mathrm{EV}$ containing media was used to harvest large EVs for each experiment as described and the 2000 $\times g$ pellets re-suspended in either (1) $2 \mathrm{~mL}$ serum-free DMEM or (2) $2 \mathrm{~mL}$ Cell Mask Orange (CMO: $2.5 \mu \mathrm{g} / \mathrm{mL}$ in serum-free DMEM) (Fisher Scientific, Leicestershire, UK; cat C10045) and both were incubated at $37^{\circ} \mathrm{C}$ for $10 \mathrm{~min}$.

\subsection{Antibody Labelling}

See Table 2 for antibody details and manufacturer information. Antibody titrations were performed for each antibody using parent cells and EVs. In all cases, the maximum recommended volume $(5 \mu \mathrm{L})$ provided the greatest fluorescence signal from the EVs. Antibody only controls (no EVs) were 
included in the ISX analysis and showed no fluorescence events above the unstained gate in each case. Both CMO labelled and unlabelled EVs preparations were washed by addition of $5 \mathrm{~mL} 1 \%$ BSA/PBS and centrifuged at $2000 \times g$ for $30 \mathrm{~min}$. The pellets were re-suspended in $700 \mu \mathrm{L} 0.2 \mu \mathrm{m}$ filtered $1 \% \mathrm{BSA} / \mathrm{PBS}$ and split into $7 \times 100 \mu \mathrm{L}$ aliquots. For each labelling combination, $5 \mu \mathrm{L}$ directly conjugated primary antibodies were added simultaneously as follows: Non-CMO labelled EVs were used for unstained, single labelled CD63 BV421, CD9 AF647, or LAMP1 AF647 and CMO FMO controls ( $\times 2)($ BV421 + AF647: both antibodies were screened). The final aliquot was used to establish EV concentration and diameter range using TRPS analysis on the qNANO. The CMO labelled EV pellet was re-suspended in $700 \mu \mathrm{L} 0.2 \mu \mathrm{m}$ filtered $1 \%$ BSA/PBS and split into $7 \times 100 \mu \mathrm{L}$ aliquots. CMO labelled EVs were used as single stained (CMO+ only), AF647 FMO (CMO + BV421), BV421 FMO (CMO + AF647), and multiplexed CMO + CD63 BV421 + either CD9 AF647 or LAMP1 AF647. Antibodies were incubated for $1 \mathrm{~h}$ on ice in the dark. EVs were washed by addition of $500 \mu \mathrm{L} 0.2 \mu \mathrm{m}$ filtered $1 \%$ BSA/PBS and centrifuged at $2000 \times g$ for $30 \mathrm{~min}$. Resulting pellets were re-suspended in $75 \mu \mathrm{L} 0.2 \mu \mathrm{m}$ filtered 1\% BSA/PBS for ISX analysis. Fully labelled EV preparations were treated post-acquisition with $0.1 \%$ Triton-X 100 and acquired again to demonstrate loss of fluorescence due to antigen degradation.

Table 2. Chemicals and Reagents Including Antibodies.

\begin{tabular}{|c|c|c|c|c|c|}
\hline \multicolumn{2}{|c|}{ Reagent } & \multicolumn{2}{|c|}{ Manufacturer } & \multicolumn{2}{|c|}{ Catalogue No. } \\
\hline \multicolumn{2}{|c|}{$\begin{array}{c}\text { Cell mask orange: plasma membrane } \\
\text { marker }\end{array}$} & \multicolumn{2}{|c|}{ Thermo Fisher Scientific } & \multicolumn{2}{|c|}{ C10045 } \\
\hline \multicolumn{2}{|c|}{ Annexin V APC/PI: Apoptosis assay } & \multicolumn{2}{|c|}{ Biolegend } & \multicolumn{2}{|c|}{640932} \\
\hline \multicolumn{2}{|c|}{$\begin{array}{l}\text { Alexa Fluor } 555 \text { phalloidin: Polymerised } \\
\text { actin cytoskeleton }\end{array}$} & \multicolumn{2}{|c|}{ Thermo Fisher Scientific } & \multicolumn{2}{|c|}{ A34055 } \\
\hline \multicolumn{2}{|c|}{$\begin{array}{l}\text { Speed beads: Imagestream flow } \\
\text { calibration }\end{array}$} & \multicolumn{2}{|c|}{ Amnis } & \multicolumn{2}{|c|}{400041} \\
\hline \multicolumn{2}{|c|}{ Quantibrite PE Beads } & \multicolumn{2}{|c|}{ Beckton Dickinson UK } & \multicolumn{2}{|c|}{340495} \\
\hline Antibody & Clone & Fluorophore & Isotype & Manufacturer & Catalogue No. \\
\hline Anti-human CD 63 & H5C6 & BV421 & Mouse IgG1, k & Biolegend & 353030 \\
\hline Anti-human CD9 & MEM-61 & AF647 & Mouse IgG1 & $\begin{array}{c}\text { Fisher } \\
\text { Scientific }\end{array}$ & 15317424 \\
\hline Anti-human LAMP 1 & H4A3 & AF647 & Mouse IgG1, $\mathrm{k}$ & Biolegend & 328611 \\
\hline
\end{tabular}

\subsection{Imagestream Acquisition}

Sheath buffer (PBS without calcium and magnesium: SIGMA, Gillingham, UK; Cat D5652) was filtered using $0.2 \mu \mathrm{m}$ bottle top filters (SIGMA, Gillingham, UK, Nalgene: FIL8184) to minimise background signal. Internal instrument calibrations were performed before every run according to manufacturer's guidelines using the ASSIST Calibrations to include: camera synchronisation, spatial offsets, dark current, bright field crosstalk coefficient, core stage position, horizontal laser, side scatter, and a retro illumination scheme to maximise the amount of light incident. This was followed by a series of internal operations designed to measure performance including excitation laser power, bright field uniformity, and focus. Specific laser powers used for this study are detailed in Table 3.

Table 3. Settings used for Imagestream.

\begin{tabular}{cccc}
\hline Laser & Channel/Filter & Power $\mathbf{m W}$ & Parameter \\
\hline 405 & Ch07/435-80 nm & 120 & BV421 \\
\hline 561 & Ch03/577-35 nm & 200 & CMO \\
\hline 785 & Ch06/762-35 nm & 70 & SSC \\
\hline 642 & Ch11/702-85 & 150 & AF647 \\
\hline
\end{tabular}


Speed beads with an exaggerated irregular surface were incorporated into every analysis for internal calibration. A dedicated laser was assigned to assess side scatter ( $\mathrm{CH}$ 06; SSC: $785 \mathrm{~nm}$ laser). For each experiment, a separate readout was obtained from $0.2 \mu \mathrm{m}$ filtered $1 \% \mathrm{BSA} / \mathrm{PBS}$ acquisition buffer alone. All events were acquired for $5 \mathrm{~min}$ and visualised using bivariate plots of side scatter against fluorescence intensity.

For cells, 10,000-30,000 total events were acquired. EVs were acquired for $5 \mathrm{~min}$ at $\times 60$ magnification using lasers as described (Table 3) (Image stream, Amnis, Seattle, WA, USA). The $\times 60$ objective provides a Numerical Aperture of 0.9 enabling resolution of $0.3 \mu \mathrm{m}^{2} /$ pixel [19].

\section{Compensation}

Antibody labelled compensation beads (anti-mouse compensation beads: BD Biosciences, San Jose, CA, USA; cat 552843) were used to acquire single colour controls within the channels used for this study. The final compensation matrices were constructed by the wizard (INSPIRE) with manual adjustment in consultation with the manufacturer's specialist adviser and applied to the .rif files of all controls, dual and triple labelled EVs. Data were analysed using the IDEAS software (v. 6.2, Amnis, Seattle, WA, USA). The compensation matrices and analysis template were applied using the batch processing tool to all .rif files to produce daf files for each sample. FCS files were exported and uploaded onto the Flow Repository according to the requirements.

\section{Mask Selection for Assessment of EV Diameter}

We investigated which of the diameter masks available within the IDEAS software would be most accurate for EVs. We used non-fluorescence size calibration beads (Fisher Scientific-UK Ltd., Loughborough, UK; cat F13838) to validate the masks. The beads were acquired using the same laser powers and settings as the EV preparations and analysis templates were constructed to identify which mask fitted most closely to the bead diameter according to the manufacturer's instructions. We found that applying the diameter mask Erode (03; indicating 3 pixel erosion) to the bright field channel most closely assigned the correct diameter. This mask formed part of an analysis template which was applied to all samples using the batch analysis tool.

\section{Molecules of Equivalent Soluble Fluorochrome (MESF) Calculation}

To enable comparisons between experiments, Molecules of Equivalent Soluble Fluorochrome (MESF) values were calculated as previously described [19,24]. Quantibrite PE beads (BD Biosciences, San Jose, CA, USA; Cat: 340495. Lot: 90926) were the closest available calibration beads for the fluorescent channel used to detect Cell Mask Orange labelled EVs. A fresh aliquot of lyophilised Quantibrite beads was reconstituted for each run, and 5000 events were acquired using the identical laser settings for each fluorophore as described. The SSC laser (channel 06) was adjusted to ensure the beads could be visualised on the bivariate plots and therefore each bead could be gated as a separate population and the median fluorescence intensity recorded. The $\mathrm{CMO}+$ events were then analysed for expression of the EV markers included in this study: CD63, CD9 and LAMP1.

\section{Discussion}

Our principal aim was to develop a standardised method for the isolation and characterisation of individual large EVs, which could be further developed for phenotyping large EVs from clinical samples. The value of the Imagestream to the field of EV characterisation has been explored elsewhere [19,24,25]. However, reports focussing on the large EV populations, frequently discarded during small EV isolation protocols, are rarely present in the current literature.

By using in vitro cultures, we were able to use the ideal conditions to generate EVs and optimise experiments. Specifically, by culturing in serum-free media, we eliminated contamination from bovine EVs present in FBS [18] and subsequent false positive fluorescence signals from serum lipoproteins [20]. Nevertheless, harvesting large EVs from any source presents challenges as cells or cell debris, including 
intracellular vesicles released due to parent cell membrane rupture from early centrifugation steps, can contaminate the subsequent EV pellets. For the EVs to be truly extracellular prior to isolation, the outer membrane of accompanying cells must not be ruptured by mechanical or chemical means during initial harvest. The centrifugation speeds we have used here are low compared to some commonly reported EV isolation protocols [26] to specifically preserve large EV membrane integrity. Electron microscopy remains the sole technique that can examine individual EVs and EV preparations for sheared cell fragments, but it is neither quantitative nor high throughput. In addition, whilst TEM is widely used for EV investigation, it cannot perhaps distinguish between bone fide EVs and other particles. In this case, cryoEM or CLEM (correlative light and electron microscopy) which facilitates overlaid fluorescence or gold labelled antibody binding and EM would prove more informative. It is necessary to use a combination of techniques to explore the quantity, quality, and biology of EVs. All techniques, many originally designed for analysing cells, have technical challenges when applied to considerably smaller entities. For flow cytometry, background scatter events due to particles in the sheath fluid are an anticipated phenomenon which is rarely reported. In the work we report here, a high level of background appeared within the same gate as unstained EVs and persisted despite $0.2 \mu \mathrm{m}$ filtration of sheath fluid. Our protocol is therefore reliant on strong and uniform, membrane-bound fluorescence labelling in order to assign an initial gate that separates potential EVs from speed beads or background scatter events. However, we found that the lower fluorescence intensity threshold to define CMO positive events was not clearly distinct from the instrument background. This was likely due to a combination of EV size and relative fluorescence intensity. As recommended elsewhere [19] we used commercially available fluorescent beads of known intensity (Quantibrite) to provide a means to assign standardised units (MESF) and therefore a mathematical cut-off for our $\mathrm{CMO}+$ gate. We acknowledge that calibration beads differ in the physical properties of EVs, in particular the refractive index, which can affect the intensity of scattered light, a particularly important consideration for the study of smaller EVs. PE was the closest available fluorophore to CMO and used as a standard for channel 03 on the ISX. $\mathrm{CMO}$ is a membrane label incorporated into the plasma membrane, and emits a greater fluorescence compared with a target-specific, conjugated antibody. This hampered the use of FITC alongside CMO as the spectrally close fluorophores led to overcompensation between channels 02 (FITC) and channel 03 (CMO). BV421 was a successful alternative but the difficulties encountered raised concerns about trying to further multiplex with additional fluorophores using this platform.

The challenges for choosing the correct technique(s) to analyse EVs have been well described elsewhere [27]. For detailed advice on considerations for EV separation or enrichment methodologies and recommended steps for EV characterisation, we recommend referring to the MISEV guidelines; a position paper prepared by the international EV community to support EV research [1]. A major challenge now is to adapt the protocol we describe for the analysis of large EVs from biological fluids. Clinical samples are more complex: EVs from a single cell type as described here offer the ideal model for characterisation, however clinical samples contain heterogeneous EVs from numerous cells [10]. EV isolation has been reported from peripheral blood with some correlations to clinical outcome in other cancers $[28,29]$. We have previously identified leukaemic EVs in patients' bone marrow plasma. This was possible using a marker which identified the cell of origin (B cell marker CD19) and understanding that the bone marrow of a leukemia patient is primarily composed of malignant $B$ cells [9]. However, surface markers for Medulloblastoma cells are less well known, rather molecular signatures define subgroups in this cancer type [30]. Investigating surface marker expression on medulloblastoma cell-derived EVs would help to develop a more rapid screening tools. The value of a liquid biopsy to diagnose brain tumours in the clinic is clear and some suitable candidates have been identified in Glioblastoma [31,32]. A next step could be to investigate EVs released by primary medulloblastoma cells although the requirements for optimal culture conditions to generate sufficient material will add additional complexity. In the case of medulloblastoma, ideally, EVs circulating in either the cerebrospinal fluid or peripheral blood for comparison to matched primary tumour-derived EVs would be of considerable interest. 
Our SOP is likely to exclude most small EVs and exosomes, expected to be present in the supernatant discarded at the final step $(2000 \times g)$. Experiments to isolate these for comparison are on-going. One classification we examined in this study was the distinction from apoptotic bodies. Demonstration of intact EV membranes, a lack of fragmented nuclei staining (DAPI) and evidence that EVs were derived from viable cells supported our assertion that the EVs analysed were not apoptotic bodies or cell debris [33]. It might be possible to further distinguish these populations using molecular profiling. Other studies suggest that these EV subgroups display distinct RNA profiles [34] an approach which is reliant on pure populations and therefore robust and meticulous isolation protocols.

Our data show that large EVs are ubiquitous and whilst absolute quantification is not yet within reach, we demonstrated similar size profiles using two independent techniques: TRPS and ISX. We have previously identified EVs of up to $6 \mu \mathrm{M}$ using immunofluorescence, ISX and TEM [15]. However, validating the quantity and size range has only been possible using the qNANO instrument. The qNANO employs TRPS technology to quantify EV count in a given sample and assign a size relative to a calibration bead of known diameter. It is currently the only instrument which can provide this information across the large EV population which spans $250 \mathrm{~nm}$ up to $6 \mu \mathrm{m}$ (from our cells). Other platforms are restricted to small EVs $(<1 \mu \mathrm{M})$ due to the measurements being reliant on Brownian movement (e.g., Nanosight and Zetaview). We found the most prevalent EV populations to be around 250-450 nm; however, we consistently detect EVs with a much larger diameter range in every cell line we have screened to date. We remain cautious not to define these as oncosomes; as although derived from cancer cells, we have not yet demonstrated their oncogenic potential [4].

Whilst fluorescence intensity alone cannot be used to quantify protein expression levels due to low level antigen expression on EVs, we did observe patterns of differential expression. In EV literature, 3 principle markers are used to define EVs: CD63, CD9, and CD81. CD63, however, has been identified as a pan-EV marker, present in all defined EV subgroups to date [6] and therefore CD63 was our preferred initial marker. However, large EVs are as yet poorly characterised and we found that CD63 was not the most abundant EV marker in our study. CD63 is a tetraspanin which has been used as a selection tool for immuno-capture experiments and also for tracking EV release [35,36]. Based on our observations, if a full EV repertoire was of interest, then a cocktail of multiple markers should be considered as screening with CD63 alone will likely fail to capture a significant proportion of EVs. We found LAMP1, previously identified on exosomes and EVs from a variety our cell types and biological fluids [37], was significantly more prevalent.

We also show here that the majority of large EVs did not express any of the three markers screened. We did observe a significant increase in the median diameter of individual EVs which expressed 1 or more markers compared to none (CMO only), in each of the experiments performed. Further, across all replicates, those EVs which co-expressed 2 markers (CD63 + CD9, or CD63 + LAMP1) were significantly larger than those without. Others have suggested that larger EVs are likely to accommodate a greater number of tumour-derived molecules than exosomes [13] and data presented here would support that hypothesis. Further investigations to define a broader panel of large EV markers followed by more comprehensive techniques, such as proteomic profiling, would help to fully phenotype the large EVs population. From a clinical perspective, it is likely that large EVs will be a rich source of biomarkers of benefit to the study of human disease. Standardised protocols and instruments capable of measuring multiple markers are key to moving the field forward and expanding the interest from exosomes only.

The research we report here demonstrates that high resolution, high throughput imaging flow cytometry is an exceptional tool offering the unique ability to quantify and analyse individual events within heterogeneous EV populations. We set out to develop an isolation protocol consisting of minimal manipulation and processing which may abrogate, mask, or indeed elicit changes in EV structure or biology, which could impact on any functional read outs in downstream experiments. Indeed, fully understanding the biological consequence(s) of EV release or uptake by recipient cells is an essential part of the field and will advance with new technologies and innovation. 
Author Contributions: Conceptualization, S.M.J. and A.B.; Data curation, S.M.J., A.B., C.S. and A.M.; Formal analysis, S.M.J., A.B. and M.G.M.; Funding acquisition, S.M.J. and M.G.M.; Investigation, S.M.J. and C.S.; Methodology, C.S. and A.M.; Resources, A.B. and A.M.; Validation, S.M.J.; Writing-original draft, S.M.J. Writing-review \& editing, A.B. and M.G.M. All authors have read and agreed to the published version of the manuscript.

Funding: This research was funded by CRUK grant number C68251/A28065.

Conflicts of Interest: The authors declare no conflict of interest.

\section{References}

1. Thery, C.; Witwer, K.W.; Aikawa, E.; Alcaraz, M.J.; Anderson, J.D.; Andriantsitohaina, R.; Antoniou, A.; Arab, T.; Archer, F.; Atkin-Smith, G.K.; et al. Minimal information for studies of extracellular vesicles 2018 (MISEV2018): A position statement of the International Society for Extracellular Vesicles and update of the MISEV2014 guidelines. J. Extracell. Vesicles 2018, 7, 1535750. [CrossRef]

2. Cocucci, E.; Meldolesi, J. Ectosomes and exosomes: Shedding the confusion between extracellular vesicles. Trends Cell Biol. 2015, 25, 364-372. [CrossRef] [PubMed]

3. Van Niel, G.; Porto-Carreiro, I.; Simoes, S.; Raposo, G. Exosomes: A common pathway for a specialized function. J. Biochem. 2006, 140, 13-21. [CrossRef] [PubMed]

4. Meehan, B.; Rak, J.; Di Vizio, D. Oncosomes-Large and small: What are they, where they came from? J. Extracell. Vesicles 2016, 5, 33109. [CrossRef] [PubMed]

5. Yanez-Mo, M.; Siljander, P.; Andreu, Z.; Zavec, A.B.; Borràs, F.E.; Buzas, E.I.; Buzas, K.; Casal, E.; Cappello, F.; Carvalho, J.; et al. Biological properties of extracellular vesicles and their physiological functions. J. Extracell. Vesicles 2015, 4, 27066. [CrossRef]

6. Kowal, J.; Arras, G.; Colombo, M.; Jouve, M.; Morath, J.P.; Primdal-Bengtson, B.; Dingli, F.; Loew, D.; Tkach, M.; Théry, C. Proteomic comparison defines novel markers to characterize heterogeneous populations of extracellular vesicle subtypes. Proc. Natl. Acad. Sci. USA 2016, 113, E968-E977. [CrossRef]

7. Simpson, R.J.; Mathivanan, S. Extracellular Microvesicles: The Need for Internationally Recognised Nomenclature and Stringent Purification Criteria. J. Proteomics Bioinform. 2012, 5. [CrossRef]

8. Tkach, M.; Thery, C. Communication by Extracellular Vesicles: Where We are and Where We Need to Go. Cell 2016, 164, 1226-1232. [CrossRef]

9. Johnson, S.M.; Dempsey, C.; Chadwick, A.; Harrison, S.; Liu, J.; Di, Y.; McGinn, O.J.; Fiorillo, M.; Sotgia, F.; Lisanti, M.P.; et al. Metabolic reprogramming of bone marrow stromal cells by leukemic extracellular vesicles in acute lymphoblastic leukemia. Blood 2016, 128, 453-456. [CrossRef]

10. Gyorgy, B.; Szabó, T.G.; Pásztói, M.; Pál, Z.; Misják, P.; Aradi, B.; László, V.; Pállinger, É; Pap, E.; Kittel, Á.; et al. Membrane vesicles, current state-of-the-art: Emerging role of extracellular vesicles. Cell. Mol. Life Sci. 2011, 68, 2667-2688. [CrossRef]

11. Colombo, M.; Raposo, G.; Thery, C. Biogenesis, secretion, and intercellular interactions of exosomes and other extracellular vesicles. Annu. Rev. Cell Dev. Biol. 2014, 30, 255-289. [CrossRef] [PubMed]

12. Xu, R.; Rai, A.; Chen, M.; Suwakulsiri, W.; Greening, D.W.; Simpson, R.J. Extracellular vesicles in cancer-Implications for future improvements in cancer care. Nat. Rev. Clin. Oncol. 2018, 15, 617-638. [CrossRef] [PubMed]

13. Minciacchi, V.R.; Freeman, M.R.; di Vizio, D. Extracellular vesicles in cancer: Exosomes, microvesicles and the emerging role of large oncosomes. Semin. Cell Dev. Biol. 2015, 40, 41-51. [CrossRef] [PubMed]

14. Morello, M.; Minciacchi, V.R.; De Candia, P.; Yang, J.; Posadas, E.; Kim, H.; Griffiths, D.; Bhowmick, N.; Chung, L.W.K.; Gandellini, P.; et al. Large oncosomes mediate intercellular transfer of functional microRNA. Cell Cycle 2013, 12, 3526-3536. [CrossRef] [PubMed]

15. Johnson, S.M.; Dempsey, C.; Parker, C.; Mironov, A.; Bradley, H.; Saha, V. Acute lymphoblastic leukaemia cells produce large extracellular vesicles containing organelles and an active cytoskeleton. J. Extracell. Vesicles 2017, 6, 1294339. [CrossRef]

16. Vagner, T.; Spinelli, C.; Minciacchi, V.R.; Balaj, L.; Zandian, M.; Conley, A.; Zijlstra, A.; Freeman, M.R.; Demichelis, F.; De, S.; et al. Large extracellular vesicles carry most of the tumour DNA circulating in prostate cancer patient plasma. J. Extracell. Vesicles 2018, 7, 1505403. [CrossRef] 
17. Pezzicoli, G.; Tucci, M.; Lovero, D.; Silvestris, F.; Porta, C.; Mannavola, F. Large Extracellular VesiclesA New Frontier of Liquid Biopsy in Oncology. Int. J. Mol. Sci. 2020, 21, 6543. [CrossRef]

18. Lehrich, B.M.; Liang, Y.; Khosravi, P.; Federoff, H.J.; Fiandaca, M.S. Fetal Bovine Serum-Derived Extracellular Vesicles Persist within Vesicle-Depleted Culture Media. Int. J. Mol. Sci. 2018, 19, 3538. [CrossRef]

19. Lannigan, J.; Erdbruegger, U. Imaging flow cytometry for the characterization of extracellular vesicles. Methods 2017, 112, 55-67. [CrossRef]

20. Simonsen, J.B. Pitfalls associated with lipophilic fluorophore staining of extracellular vesicles for uptake studies. J. Extracell. Vesicles 2019, 8, 1582237. [CrossRef]

21. Osteikoetxea, X.; Benke, M.; Rodriguez, M.; Pálóczi, K.; Sódar, B.W.; Szvicsek, Z.; Szabó-Taylor, K.; Vukman, K.V.; Kittel, Á.; Wiener, Z.; et al. Detection and proteomic characterization of extracellular vesicles in human pancreatic juice. Biochem. Biophys. Res. Commun. 2018, 499, 37-43. [CrossRef] [PubMed]

22. Consortium, E.-T.; Van Deun, J.; Mestdagh, P.; Agostinis, P.; Akay, Ö.; Anand, S.; Anckaert, J.; Martinez, Z.A.; Baetens, T.; Beghein, E.; et al. EV-TRACK: Transparent reporting and centralizing knowledge in extracellular vesicle research. Nat. Methods 2017, 14, 228-232.

23. Vogel, R.; Coumans, F.A.W.; Maltesen, R.G.; Böing, A.N.; Bonnington, K.E.; Broekman, M.L.; Broom, M.F.; Buzás, E.I.; Christiansen, G.; Hajji, N.; et al. A standardized method to determine the concentration of extracellular vesicles using tunable resistive pulse sensing. J. Extracell. Vesicles 2016, 5, 31242. [CrossRef] [PubMed]

24. Gorgens, A.; Bremer, M.; Ferrer-Tur, R.; Murke, F.; Tertel, T.; Horn, P.A.; Thalmann, S.; Welsh, J.A.; Probst, C.; Guerin, C.; et al. Optimisation of imaging flow cytometry for the analysis of single extracellular vesicles by using fluorescence-tagged vesicles as biological reference material. J. Extracell. Vesicles 2019, 8, 1587567. [CrossRef] [PubMed]

25. Headland, S.E.; Jones, H.R.; D'Sa, A.S.V.; Perretti, M.; Norling, L.V. Cutting-edge analysis of extracellular microparticles using Image Stream (X) imaging flow cytometry. Sci. Rep. 2014, 4, 5237. [CrossRef] [PubMed]

26. Witwer, K.W.; Buzás, E.I.; Bemis, L.T.; Bora, A.; Lässer, C.; Lötvall, J.; Hoen, E.N.N.T.; Piper, M.G.; Sivaraman, S.; Skog, J.; et al. Standardization of sample collection, isolation and analysis methods in extracellular vesicle research. J. Extracell. Vesicles 2013, 2, 2. [CrossRef] [PubMed]

27. Erdbrugger, U.; Lannigan, J. Analytical challenges of extracellular vesicle detection: A comparison of different techniques. Cytom. Part A 2016, 89, 123-134. [CrossRef]

28. Menck, K.; Bleckmann, A.; Wachter, A.; Hennies, B.; Ries, L.; Schulz, M.; Balkenhol, M.; Pukrop, T.; Schatlo, B.; Rost, U.; et al. Characterisation of tumour-derived microvesicles in cancer patients' blood and correlation with clinical outcome. J. Extracell. Vesicles 2017, 6, 1340745. [CrossRef]

29. Taylor, D.D.; Gercel-Taylor, C. MicroRNA signatures of tumor-derived exosomes as diagnostic biomarkers of ovarian cancer. Gynecol. Oncol. 2008, 110, 13-21. [CrossRef]

30. Juraschka, K.; Taylor, M.D. Medulloblastoma in the age of molecular subgroups: A review. J. Neurosurg. Pediatr. 2019, 24, 353-363. [CrossRef]

31. Santiago-Dieppa, D.R.; Steinberg, J.; Gonda, D.; Cheung, V.J.; Carter, B.S.; Chen, C.C. Extracellular vesicles as a platform for 'liquid biopsy' in glioblastoma patients. Expert Rev. Mol. Diagn. 2014, 14, 819-825. [CrossRef] [PubMed]

32. Saenz-Antonanzas, A.; Auzmendi-Iriarte, J.; Carrasco-Garcia, E.; Moreno-Cugnon, L.; Ruiz, I.; Villanua, J.; Egaña, L.; Otaegui, D.; Samprón, N.; Matheu, A. Liquid Biopsy in Glioblastoma: Opportunities, Applications and Challenges. Cancers (Basel) 2019, 11, 950. [CrossRef] [PubMed]

33. Ihara, T.; Yamamoto, T.; Sugamata, M.; Okumura, H.; Ueno, Y. The process of ultrastructural changes from nuclei to apoptotic body. Virchows Arch. 1998, 433, 443-447. [CrossRef] [PubMed]

34. Crescitelli, R.; Lässer, C.; Szabó, T.G.; Kittel, A.; Eldh, M.; Dianzani, I.; Buzás, E.I.; Lötvall, J. Distinct RNA profiles in subpopulations of extracellular vesicles: Apoptotic bodies, microvesicles and exosomes. J. Extracell. Vesicles 2013, 2, 2. [CrossRef]

35. Verweij, F.J.; Bebelman, M.P.; Jimenez, C.R.; Garcia-Vallejo, J.J.; Janssen, H.; Neefjes, J.; Knol, J.C.; Haas, R.D.G.D.; Piersma, S.R.; Baglio, S.R.; et al. Correction: Quantifying exosome secretion from single cells reveals a modulatory role for GPCR signaling. J. Cell. Biol. 2018, 217, 1157. [CrossRef]

36. Cashikar, A.G.; Hanson, P.I. A cell-based assay for CD63-containing extracellular vesicles. PLoS ONE 2019, 14, e0220007. [CrossRef] 
37. Kalra, H.; Simpson, R.J.; Ji, H.; Aikawa, E.; Altevogt, P.; Askenase, P.W.; Bond, V.C.; Borràs, F.E.; Breakefield, X.O.; Budnik, V.; et al. Vesiclepedia: A compendium for extracellular vesicles with continuous community annotation. PLoS Biol. 2012, 10, e1001450. [CrossRef]

Publisher's Note: MDPI stays neutral with regard to jurisdictional claims in published maps and institutional affiliations.

(C) 2020 by the authors. Licensee MDPI, Basel, Switzerland. This article is an open access article distributed under the terms and conditions of the Creative Commons Attribution (CC BY) license (http://creativecommons.org/licenses/by/4.0/). 